\title{
L'analyse des organisations : un regard sur la production de langue française contemporaine (1950-1990)
}

\section{Jean-François Chanlat}

Numéro 18-19, 1992

Entreprises : approches théoriques et études de cas

URI : https://id.erudit.org/iderudit/1002305ar

DOI : https://doi.org/10.7202/1002305ar

Aller au sommaire du numéro

\section{Éditeur(s)}

Département de sociologie - Université du Québec à Montréal

ISSN

0831-1048 (imprimé)

1923-5771 (numérique)

Découvrir la revue

Citer cet article

Chanlat, J.-F. (1992). L'analyse des organisations : un regard sur la production de langue française contemporaine (1950-1990). Cahiers de recherche sociologique, (18-19), 93-138. https://doi.org/10.7202/1002305ar
Résumé de l'article

Depuis l'entre-deux-guerres où l'on voit apparaître les premières études américaines sur la question, notamment celles d'Elton Mayo, l'intérêt pour les organisations n'a fait que croître dans la plupart des pays industrialisés. Dans les pays anglophones et plus particulièrement aux États-Unis, berceau des premiers travaux dans le domaine, l'analyse des organisations est devenue, dès le début des années soixante, un champ à part entière. La croissance économique, la prolifération des organisations et leur développement, « la rationalisation du monde " ont en effet amené de plus en plus de chercheurs occidentaux à tenter de comprendre la dynamique sociale des ensembles organisés. Au cours des dernières années, cet intérêt a été renforcé par l'échec des solutions collectivistes, l'engouement pour l'entreprise et la gestion que connaît, de nos jours, l'Occident, et plus généralement par l'importance de penser le monde en termes d'organisation. Par rapport à cette tendance générale, que l'on peut aisément relever un peu partout dans les pays industrialisés, l'objet de cet article sera de montrer comment le champ francophone s'est développé et comment il se distingue encore aujourd'hui du champ anglo-saxon, notamment de l'analyse des organisations telle qu'elle se pratique majoritairement en Amérique du Nord.
Ce document est protégé par la loi sur le droit d'auteur. L'utilisation des services d’Érudit (y compris la reproduction) est assujettie à sa politique d'utilisation que vous pouvez consulter en ligne.

https://apropos.erudit.org/fr/usagers/politique-dutilisation/ 


\section{L'analyse des organisations: un regard sur la production de langue française contemporaine (1950-1990)}

Depuis l'entre-deux-guerres, où l'on voit apparaître les premières études américaines sur la question ${ }^{1}$, notamment celles d'Elton Mayo (Desmarez, 1986), l'intérêt pour les organisations n'a fait que croître dans la plupart des pays industrialisés. Dans les pays anglophones et plus particulièrement aux États-Unis, berceau des premiers travaux dans le domaine, l'analyse des organisations est devenue, dès le début des années soixante, un champ à part entière (Morgan, 1989; Reed, 1985; Séguin et Chanlat, 1988; Chanlat et Séguin, 1987; Ballé, 1990).

La croissance économique, la prolifération des organisations et leur développement, "la rationalisation du monde" ont en effet amené de plus en plus de chercheurs occidentaux à tenter de comprendre la dynamique sociale des ensembles organisés. Au cours des dernières années, cet intérêt a été renforcé par l'échec des solutions collectivistes, l'engouement pour l'entreprise et la gestion que connaît l'Occident, et plus généralement par l'importance de penser le monde en termes d'organisation (Morin, 1986). Par rapport à cette tendance générale, que l'on peut aisément relever un peu partout dans les pays industrialisés, l'objet de cet article sera, d'une part, de rappeler à partir d'une étude des principaux travaux comment le champ francophone ${ }^{2}$ s'est développé et, d'autre part, d'avancer quelques hypothèses

\footnotetext{
- L'auteur tient à remercier tout particulèrement Gilles Amado, Catherine Ballé, Richard Déry, Alain Eraly, Eugène Enriquez, John Hassard, Dany Miller, Thierry Pauchan et Didier Van den Hove pour leurs commentaires judicieux concernant une première version de ce texte. La version finale n'engage que la seule responsabilité de l'auteur.

1 Compte tenu de la nature de la contribution de Jean-François Chanlat, c'est-à-dire une revue exhaustive de la littérature française sur la question des organisations, nous avons pensé qu'il était nécessaire, contrairement à notre présentation habituelle, d'inclure les références bibliographiques directement dans le texte.

2 Par francophone, nous entendons ici tous les pays de langue française. Si notre analyse porte principalement sur deux champs régionaux particuliers, la France et le Québec, elle tente dans la mesure du possible de rendre compte à l'occasion de certains travaux menés dans d'autres pays de langue française.
} 
concernant son originalité par rapport au champ anglo-saxon ${ }^{3}$, notamment américain.

Trop souvent méconnue pour ne pas dire ignorée par les chercheurs de langue anglaise, en particulier nord-américains, lanalyse en langue française des organisations mérite par sa diversité et son originalité de sortir des frontières linguistiques à l'intérieur desquelles elle a été malheureusement jusqu'ici confinée. ${ }^{4}$

\section{De la sociologie industrielle à l'analyse des organisations: plus de quatante ans de recherche}

Dans les pays de langue française, les premières interrogations sociologiques touchant les organisations sont issues des travaux du sociologue Georges Friedmann. En stattachant aux problèmes humains du machinisme industriel (1947), à la division du travail (1956), à la technique (1966), l'auteur de Où va le travail humain? (1950), tout en faisant la critique des travaux de la sociologie industrielle américaine menés à cette époque, jette, après la Deuxième Guerre mondiale, les premiers jalons de la sociologie du travail de langue française (Friedmann et Naville, 1961).

Pour Friedmann, la sociologie du travail se définit comme "l'étude, sous leurs divers aspects, de toutes les collectivités humaines qui se constituent à l'occasion du travail" (1961, p. 26). C'est à l'intérieur de ce vaste cadre qu'il situe la sociologie des organisations. Cette demière n'est qu'une des approches privilégiées des collectivités de travail. Si Friedmann semble favorable à des recherches de ce genre, initiées par les chercheurs anglophones dont il connaît les travaux, il reste que la plupart des recherches de cette sociologie du travail en construction porteront sur les rapports de travail, les divers facteurs qui les affectent et les enjeux sociopolitiques qui en découlent (Gorz, 1973, 1988: Actes, 1978). Autrement dit, durant de nombreuses années, la sociologie du travail fera office (pour la plupart des sociologues français) de sociologie des organisations.

L'organisation en tant qu'objet central d'étude n'émergera, en effet, véritablement dans les pays de langue française qu'au tournant des années 60 . C'est autour de Michel Crozier, qui deviendra fort célèbre par la suite, que se constitue un

3 Par champ anglo-saxon, nous entendons la production réalisée par des auteurs dont la principale langue d'usage est l'anglais. Mais, comme il existe d'importantes différences entre la tradition britannique et la tradition américaine, nous préciserons à l'occasion ces différences.

4 À l'exception de M. Rose $(1977,1987)$ qui a largement fait état des travaux de la sociologie industrielle française et l'article récent de Cornelius Lammers (1990), peu d'auteurs de langue anglaise mentionnent les travaux de langue française. Le seul auteur français qui a su franchir la barrière du silence est bien sûr Michel Crozier. Nous reviendrons un peu plus loin sur cette ignorance qui est d'ailleurs souvent réciproque. 
des premiers groupes sinon le premier voué à la recherche sur le sujet. Fondé en 1962, le centre de sociologie des organisations (CSO) a pour objectif d'étudier les institutions françaises dans un contexte de changement social rapide. Ce désir de comprendre la dynamique des organisations est nouveau dans le paysage français de l'époque. Contrairement aux chercheurs de langue anglaise qui consacrent au même moment la sociologie des organisations comme un champ à part entière de recherche (Etzioni, 1961; Blau et Scott, 1962), les chercheurs de langue française, pour des raisons linguistiques mais aussi et surtout pour des raisons socioculturelles, se désintéressent des études anglo-saxonnes déjà publiées dans ce domaine. Ce n'est pas le cas du CSO, le principal centre de recherche. Contrairement aux sociologues qui les environnent, ses membres organisent des séminaires sur les publications anglo-saxonnes et plusieurs chercheurs américains célèbres séjournent en son sein, notamment Aiken, Hage, Kimberly et Hirschman.

Plus tournés vers la théorie, l'étude des grandes questions sociales, la plupart des sociologues français, largement inspirés par le marxisme, ont en effet tendance à voir dans ces travaux, quand ils les connaissent, une sociologie industrielle appliquée au service d'intérêts patronaux (Mottez, 1971; Ballé, 1990). Les premiers travaux de Michel Crozier seront d'ailleurs longtemps considérés par ses collègues comme relevant de la tradition américaine et donc jugés de la même manière (Ballé, 1990).

C'est donc dans un milieu sociologique relativement hostile à ce genre de démarche que Michel Crozier fonde son centre de recherche. Il fait auvre de pionnier à un double titre. D'une part, dans le domaine de la recherche proprement dite, un de ses ouvrages, Le phénomène bureaucratique, publié en 1963, constituera pendant longtemps une remarquable exception dans la production sociologique de langue française. D'autre part, sur le plan de la formation des chercheurs, son centre constituera durant de nombreuses années un des lieux privilégiés d'apprentissage pour tous ceux qui s'intéressent à la dynamique organisationnelle ${ }^{5}$.

Inspiré par les travaux américains menés au cours des années cinquante, notamment par Gouldner, Merton, Selznick, et March et Simon, et guidé par un objectif, à savoir la compréhension des organisations françaises, les premiers travaux de ce nouveau centre de recherche se penchent sur un certain nombre de thèmes: l'introduction de l'informatique dans l'entreprise (Ballé et Peaucelle, 1972), l'analyse d'un conflit industriel en mai 1968 (Kergoat, 1973), l'étude d'un ministère (Thoenig, 1973), l'administration française (Crozier, Friedberg, Grémion et Grémion, 1979), la réforme régionale (Grémion, 1976), le travail ouvrier (Sainsaulieu, 1972). Si pendant un certain nombre d'années, le CSO constituera l'un des principaux pôles de recherche francophone, s'attachant à analyser surtout la modernisation des institutions publiques françaises, les années soixante-dix et

5 À cet égard, lorsqu'on jette un oeil sur la liste des personnes qui sont passés par le $\mathrm{CSO}$, on constate qu'un grand nombre de chercheurs contemporains en ont fait partie à un moment donné ou à un autre. 
quatre-vingt vont voir émerger d'autres lieux de recherche, à la fois en France et hors de l'Hexagone, notamment au Québec.

En France, l'introduction de nouveaux enseignements à la fois dans les grandes écoles françaises et à l'université, la création de nouveaux centres de recherche affiliés ou non au CNRS ${ }^{6}$, l'intérêt croissant des laboratoires de sociologie du travail pour l'organisation ${ }^{7}$ vont peu à peu élargir et stimuler, à la fin des années soixante-dix, les travaux dans ce domaine. À l'extérieur des frontières françaises, on observe un phénomène similaire, notamment au Québec où les grandes réformes politiques, sociales et éducatives réalisées, au cours des années soixante et soixante-dix, encouragent les recherches organisationnelles ${ }^{8}$.

Contrairement à la situation française où l'analyse des organisations est encore à cette époque un objet d'étude en quête de sa légitimité et où les travaux de langue anglaise ne sont connus que d'une poignée de chercheurs (Ballé, 1990), la proximité des États-Unis et les liens avec le Canada anglais ne provoquent pas les mêmes réactions dans l'univers québécois à l'exception des départements de sociologie.

En effet, dans ces départements, et ce même si on y enseigne officiellement la sociologie des organisations, la position de ce type d'enseignement s'apparente à ce qu'on observe dans le paysage sociologique français. À part la question nationale qui structure à l'époque bon nombre de recherches et de débats, les points de vue théoriques et méthodologiques des sociologues québécois en matière d'organisation se rapprochent plus de ceux exprimés par leurs homologues français que de ceux défendus par les sociologues américains, notamment les structuro-fonctionnalistes.

\footnotetext{
6 Les principaux étant le Centre de recherche en gestion de l'École polytechnique, le Centre de gestion scientifique et le Laboratoire de sociologie de l'innovation de l'École de Mines, le Laboratoire de sociologie du changement des Institutions (CNRS), le Laboratoire du Cnam, le Glysi à Lyon et le Lest à Aix en Provence. Voir notamment l'article de M. Berry (1986).

7 Le numéro spécial de la revue Sociologie du travail, no 3, 1986 "Retour vers l'entreprise" en est une bonne et récente illustration.

8 Toutes les écoles et facultés de gestion québécoises, quelles que soient leur particularité (administration privée, publique, scolaire, hospitalière, etc.), voire les Ecoles d'ingénieurs intègrent d'une manière ou d'une autre dans leurs programmes un ou plusieurs cours concernant les organisations. C'est ainsi, par exemple, qu'il existe un enseignement structuré et obligatoire de la sociologie des organisations à l'École des hautes études commerciales de Montréal depuis 1968. Sous des appellations parfois différentes, on peut observer un enseignement analogue à l'ENAP, l'École polytechnique de Montréal, la faculté des sciences de l'administration de l'Université Laval, ou dans les départements des sciences administratives du réseau de l'Université du Québec. Ce type d'enseignement se retrouve également dans les départements de science politique, sociologie, communications, etc.
} 
La communauté de langue, la formation française de nombreux sociologues québécois ${ }^{9}$, le caractère distinct du Québec ne sont pas étrangers à cette situation.

Si les années soixante et soixante-dix peuvent être considérées comme le temps de l'émergence des premiers travaux de langue française, les années quatrevingt vont être le théâtre d'un intérêt renouvelé envers les phénomènes organisationnels. Ce phénomène sera d'autant plus perceptible en France que le milieu sociologique était demeuré, malgré quelques îlots, assez réfractaire à ce genre d'analyse. Le culte de l'entreprise (Rousseau, 1988), le déclin de l'orthodoxie marxiste (Gorz, 1988), l'omniprésence des organisations et des entreprises (Sainsaulieu, 1987), les difficultés des services publiques, la crise de l'État providence (Rosanvallon, 1981), la question syndicale (Rosanvallon, 1988), l'échec des pays de l'Est et la faillite des grands systèmes théoriques contribuent, chacun à sa manière, à légitimer les analyses organisationnelles. C'est un peu comme si l'intérêt qui animait la poignée de chercheurs regroupés autour de Michel Crozier au milieu des années soixante, avait peu à peu gagné d'autres secteurs de la recherche française (Ballé, 1990).

Au Québec, ce phénomène est également observable, par exemple, dans les départements de sociologie où l'organisation et l'entreprise sont devenues des objets de recherche à part entière et dans les départements de communication où l'on assiste à un engouement pour les phénomènes organisationnels (Taylor, 1988). Plus généralement, on y note aussi ce que l'on retrouve dans les pays de langue anglaise, à savoir une migration des chercheurs en sciences sociales vers les écoles et facultés de gestion (Hinings, 1988; Lorsch, 1987). La popularité des études en sciences administratives, l'intérêt croissant des écoles et facultés de gestion pour les sciences humaines ouvrent en effet des portes aux sociologues, voire aux anthropologues. Ces ouvertures sont d'autant plus importantes que les départements de sociologie n'offrent plus les mêmes opportunités en matière d'emploi, à moins qu'ils n'aient eux-mêmes développé des recherches dans les domaines qui sont de nos jours en expansion (santé, entreprise, science et technologie, environnement, etc.) (Fournier, Trépanier et Girard, 1985).

Les quarante dernières années ont donc été marquées par de profonds changements. Aux premiers travaux accomplis en France dès l'après-guerre par Georges Friedmann en résonance à la sociologie industrielle anglo-américaine et à ceux de Michel Crozier et son équipe amorcés un peu plus tard dans un climat intellectuel défavorable ont succédé des recherches de plus en plus nombreuses et diversifiées dans la plupart des pays de langue française, notamment en France et au Québec. Si une certaine méconnaissance, voire ignorance des travaux anglophones se fait encore sentir dans certains milieux de l'Hexagone, ce qui est, bien sûr, beaucoup moins le cas au Québec, on peut affirmer sans aucun doute que l'étude

9 Dans les années soixante-dix, deux grands courants de pensée français inspirent un bon nombre de sociologues québécois: l'actionnalisme d'Alain Touraine (1973) et les différentes composantes du marxisme français (Althusser, Poulantzas, etc.). 
des phénomènes organisationnels est aujourd'hui devenue parfaitement légitime dans les pays de langue française, notamment pour les sciences humaines.

Ce bref rappel historique nous amène à faire deux grandes constatations. La première concerne l'éclatement des recherches qu'a connu ces dernières années ce champ d'étude. La seconde touche l'originalité du champ francophone. Ce sont ces deux aspects que nous aborderons maintenant.

\section{L'analyse des organisations de langue française: un champ très diversifié}

Depuis les premiers travaux d'inspiration friedmannienne dans les années cinquante et surtout ceux de Crozier et son équipe dans les années soixante, le champ de l'analyse organisationnelle a beaucoup changé. La sociologie qui avait constitué le noyau disciplinaire de départ a été peu à peu rejointe par d'autres disciplines (psycho-sociologie, sciences politiques, anthropologie, psychanalyse, socio-linguistique, épistémologie, communication). À l'instar des pays anglophones, qui ont connu un éclatement similaire des recherches et des paradigmes (Burrell et Morgan, 1979; Reed, 1985; Morgan, 1989; Hassard et Pym, 1990), le champ francophone apparait, de nos jours, comme un "bazar théorique et méthodologique". En effet, à l'intérieur de ce champ, on retrouve un certain nombre de courants qui puisent à des disciplines diverses, font appel à des paradigmes différents, et originent de lieux fort variés. Les courants les plus notables sont les suivants: 1) le courant de l'analyse stratégique; 2) le courant de l'analyse socio-culturelle; 3) le courant psycho-sociologique; 4) le courant actionnaliste; 5) le courant systémique; 6) le courant socio-politique; 7) le courant "anthropologique"; 8) le courant communicationnel; 9) le courant épistémologique $^{10}$.

Le courant de l'analyse stratégique

Ce courant est directement issu des travaux de Michel Crozier (1963) et Erhard Friedberg (1972). Il part du principe que tout individu en situation de travail n'est jamais totalement déterminé dans son comportement (Crozier et Friedberg, 1977). S'opposant au déterminisme social absolu, qu'il soit de nature marxiste ou autre ${ }^{11}$, l'analyse stratégique postule, comme le rappelle fort justement Bernoux (1985), qu'il n'existe pas une rationalité mais des rationalités propres à chaque acteur, que celui-ci possède une liberté relative à l'intérieur du cadre organisationnel et qu'enfin

10 Pour des raisons d'espace, nous avons mis de côté dans une large mesure les recherches en stratégie d'entreprise et les travaux d'orientation très managériale. Ils nécessiteraient un article à part.

11 À cet égard, les travaux de M. Crozier et de son équipe ne sont pas sans avoir quelque parenté avec l'individualisme méthodologique d'un Raymond Boudon (1977). 
la régulation de ces libertés individuelles ou collectives se fait au moyen du pouvoir. Chaque acteur cherchera donc à optimiser sa stratégie pour être le plus autonome possible. L'avantage comparé d'un acteur sur un autre originera des sources de pouvoir sur lesquelles il peut tabler: compétence professionnelle, maîtrise des relations avec l'environnement, information, utilisation des règles organisationnelles (Crozier et Friedberg, 1977). Cet avantage sera d'autant plus fort que l'acteur concerné maîtrisera une zone d'incertitude pertinente par rapport à la relation qu'il a engagée avec d'autres partenaires. Le courant de l'analyse stratégique voit donc l'organisation comme un construit social composé d'acteurs qui développent des stratégies singulières. En éternel mouvement, l'organisation devient un véritable système d'action concret, un champ où se déploient les différentes stratégies.

Le système social est ainsi un ensemble interdépendant de contraintes et de stratégies qui structure fortement les modes de rapports entre membres de l'organisation. Du fait qu'ils sont mis en relations obligées avec d'autres pour produire, travailleurs et cadres doivent entrer dans le jeu des autres ou s'en protéger mais ils ne peuvent rester hors-jeu, sous peine d'être ramenés à un simple élément du décor. Avec l'analyse des jeux, marges de liberté, modalités d'exercice de l'influence, et stratégies d'action, on découvre que le système social n'est pas un simple conglomérat d'éléments matériels et humains, pas plus qu'il ne se réduit aux seules règles de conduites édictées par l'organigramme et les règlements formels de travail. Le système social est une reconstitution permanente de jeux par l'effet de conduites stratégiques sans cesse reprises (Sainsaulieu, 1987, p. 118).

Ce type d'analyse, qui a émergé tout d'abord de recherches portant sur des entreprises ou des administrations publiques (Crozier, 1963, 1965; Thoenig, 1973; Crozier et Coll., 1979; Grémion, 1976; Denis-Grenier, 1974), a par la suite inspiré d'autres études portant sur le pouvoir informatique dans l'entreprise (Ballé et Peaucelle, 1972), les relations de travail dans les usines (Sainsaulieu, 1972; Piotet, Exiga et Sainsaulieu, 1981; Bernoux, 1981), les décisions administratives (Grémion, 1979), la technologie informatique (Pavé, 1989), l'entreprise (Bernoux, 1985), les universités (Friedberg et Musselin, 1989), les mairies (Dion, 1987), le changement organisationnel (Raimbault et Saussois, 1983), la technologie (Denis, 1987).

S'appuyant sur des études fouillées de cas et sur une conception pluraliste du pouvoir (Lukes, 1974), l'analyse stratégique a permis de mettre en relief à la fois l'autonomie relative des acteurs sociaux et la dynamique politique propre à chaque construit organisationnel. Même si cette perspective ne cherche pas à être macroscopique, elle développe néanmoins un point de vue systémique. Les acteurs doivent être resitués dans des ensembles organisés plus ou moins larges (Crozier et Thoenig, 1976). L'analyse de l'industrie nationale des appareils électroménagers développée par Dupuy et Thoenig (1986) est une bonne illustration de cette approche plus macroscopique de la dynamique organisationnelle. 
L'analyse stratégique, telle qu'elle s'incarne dans l'œuvre de Michel Crozier et de ses principaux disciples, a eu un rôle historique considérable dans le développement de la sociologie des organisations de langue française. En focalisant sur les acteurs dans un ensemble organisé, elle a mis en évidence certaines logiques sous-jacentes, notamment le jeu politique. Toutefois, c'est à d'autres courants que l'on doit d'avoir éclairé certaines dimensions de la dynamique humaine dans les organisations, notamment les phénomènes d'ordre culturel et la constitution affective du lien social.

\section{Le courant de l'analyse socio-culturelle}

Si l'on doit également à Michel Crozier une des premières tentatives de relier les phénomènes organisationnels à certains traits culturels propres à une société $(1963,1970)^{12}$, ce n'est qu'à la fin des années soixante-dix que l'on voit apparaître un ensemble de travaux qui s'intéressent d'abord et avant tout aux phénomènes culturels. Cet ensemble peut se diviser en deux grands groupes: le premier que je qualifierais de "managérial" et le second de socio-anthropologique.

Le courant managérial, comme son nom l'indique, est issu des écoles de gestion. Il rassemble des chercheurs dont l'objectif premier est de considérer la culture comme un instrument de gestion. S'inscrivant dans la mouvance des travaux de langue anglaise, il cherche à voir comment la culture de l'entreprise ou la culture de l'organisation affecte sa dynamique et ses résultats (Archer et Sérieyx, 1984; Allaire et Firsirotu, 1988; Abravanel et Coll., 1988; Thevenet, 1986; Lemaître, 1985, 1986). Parallèlement, le courant socio-anthropologique s'intéresse davantage à restituer l'univers culturel des organisations dans toute sa complexité. Opposé à une vision uniquement instrumentale de la culture et au caractère manipulateur qui peut y être associé (Larçon et Reitter, 1979; Bosche, 1989; Nizard, 1984; Mothé-Gautrat, 1986; Amado, 1987; Aktouf, 1989, 1990; Symons, 1988), il cherche à rendre compte de la régulation culturelle propre à chaque construit organisationnel. C'est en particulier tout le sens des travaux de Renaud Sainsaulieu et son équipe depuis une quinzaine d'années $(1977,1987)$. Leur observation des relations de travail dans un certain nombre d'ateliers et de services administratifs français les a amenés à dégager quatre modèles culturels: un modèle fusionnel où les membres d'un groupe font corps de façon très affective, un modèle de négociation et d'acceptation des différences que l'on retrouve chez les gens de métiers, un modèle marqué par les affinités sélectives que l'on retrouve chez les

12 L'analyse du phénomène bureaucratique se termine en effet par une réflexion sur le caractère singulier de la bureaucratie française qui fait une large place à la culture et à l'histoire (1963) et le sous-titre de l'ouvrage est particulièrement explicite à cet égard: "Essai sur les tendances bureaucratiques des systèmes d'organisation moderne et sur leurs relations en France avec le système social et culturel". Voir également, le chapitre "Organisation et Culture" dans Crozier et Freidberg (1977) et son livre sur les ÉtatsUnis (1980). 
autodidactes et un modèle de retrait que l'on retrouve chez les gens qui n'investissent pas dans leur travail. Cette analyse culturelle des comportements sociaux démontre que l'organisation est le lieu d'où émergent des identités collectives clairement observables et que l'entreprise est peut être devenue un des derniers lieux d'intégration sociale contemporain (Sainsaulieu, 1990). D'autres travaux vont mettre l'accent sur des éléments culturels externes. C'est le cas de l'étude de Denis Segrestin et de son étude sur les corporations où il montre combien la logique des corps de métiers est une défense de l'identité socioprofessionnelle et combien la dynamique communautaire de ces professions influe non seulement sur la dynamique des organisations mais aussi sur celle de la société globale (1985). C'est le cas également de Guy Minguet qui explique une partie du dynamisme d'une ville de l'ouest de la France: Cholet par sa tradition socioculturelle de lutte (1985), ou de Alain Chanlat qui voit dans le développement et les succès d'Hydro-Québec un élément qui a contribué à la transformation de l'identité québécoise (1984), ou encore de Jean-Pierre Dupuis qui montre comment la dynamique sociale de deux villages du Nord-Ouest québécois est marquée par les entreprises qui en ont été les fondatrices (1990).

D'autres recherches vont s'intéresser davantage aux perturbations que les changements techniques vont produire sur l'univers socio-culturel des mineurs (Moscovici, 1963; Akerman, 1970), des employés du métro parisien (Bouvier, 1985), des cheminots (Ribeil, 1984), des ouvriers et employés (Alter, 1985), des membres d'équipes semi- autonomes de production (Liu, 1982) ou encore à l'importance des règles dans la dynamique sociale de l'entreprise (Reynaud, 1989).

D'autres encore vont s'attarder à faire de véritables enquêtes ethnographiques. On n'a qu'à citer les travaux de certains membres du groupe d'ethnographie des organisations du CRG et du CGS animé par Michel Berry (1986), C. Riveline (1983) et Michel Matheu (1983), les travaux de Latour et Woolgar sur la vie dans les laboratoires (1988), ceux de Aktouf concernant l'univers ouvrier des brasseries algérienne et québécoise (1986), de Bouchard sur les camionneurs du grand nord québécois (1985), de Bertaux et Bertaux-Wiame sur l'univers des boulangers français (1981), de Linhart sur les travailleurs d'usine automobile (1978), de Bernier sur le modèle japonais d'organisation (1979), ou de Bouvier sur l'univers des conducteurs de métro (1980), l'étude ethnologique menée par Etchegoyen (1990), ou encore les études d'anthropologie industrielle de Althabe, Légé et Sélim (1984). Par ailleurs, certains auteurs francophones vont plus particulièrement s'attarder à analyser de plus près les pratiques langagières et l'exercice de la parole dans les organisations. C'est le cas de Jacques Girin (1982, 1990), d'Alain Chanlat, (1984, 1990), d'Omar Aktouf (1986), de Vacquin (1986), ou de R. Laufer et C. Paradeise qui se sont intéressés plus particulièrement à la rhétorique dans les entreprises (1981) ou encore de la tentative de Legendre de décrypter les discours sous-tendant le pouvoir managérial (1983) et plus généralement encore du groupe français qui a organisé et animé le colloque international "Travail et pratiques langagières", tenu à Paris au printemps 1989 (Actes, 1989). Enfin, d'autres chercheurs ont voulu rendre compte des différences nationales en matière 
d'organisation. Outre Crozier que nous avons déjà mentionné, on peut signaler entre autres l'important travail réalisé par Maurice, Sellier et Silvestre sur les différences sociétales France-Allemagne en matière d'éducation et de marché du travail qui affectent la dynamique des entreprises de ces deux pays (1982), l'analyse comparative, très fouillée, de Jean-Gustave Padioleau portant sur Le Monde et le Washington Post (1985), les études d'André Laurent (1983) sur la diversité culturelle des conceptions en gestion, celles de Laurent, Faucheux et Amado sur les contrastes entre la France et les États-Unis en matière de changement organisationnel (1990), celle d'Amado sur les racines culturelles des comportements organisationnels brésiliens (1990), les recherches récentes de d'Iribarne sur la logique culturelle d'entreprises nationales, notamment, françaises, hollandaises et américaines $(1986,1987,1989)$, celle de Caillies sur la confrontation franco-japonaise (1986), la comparaison d'une université allemande et française réalisée par Friedberg et Musselin (1989), l'analyse d'Alain Chanlat et R. Bédard sur l'originalité du modèle québécois d'organisation (1989), les travaux d'Harold Bhérer sur le management soviétique (1982) ou ceux de Bourgoin (1984) et de Kamdem $(1985,1990)$ sur l'entreprise africaine.

Le courant de l'analyse psycho-sociologique

$\mathrm{Si}$, comme nous venons de le voir, les travaux francophones sur les phénomènes culturels sont relativement nombreux et diversifiés, ceux qui se sont intéressés à la constitution affective du lien social et aux différentes relations qui unissent l'individu à l'organisation sont également fort nombreux. Dans la tradition francophone, on retrouve depuis plus d'une vingtaine d'années, un certain nombre de chercheurs qui, en s'appuyant à la fois sur les grandes ouvres psychologiques, notamment celle de Freud, et sur les sciences sociales ont tenté de rendre compte de l'interaction entre le psychisme et le social dans des ensembles organisés.

Un des premiers groupes sinon le premier s'est constitué autour de l'Association pour la recherche et l'intervention psycho-sociologique, ARIP, et de la revue Connexions. Fondé en 1959 sur une idée de Max Pagès et de Jean Palmade, ce groupe, auquel étaient associés à ses débuts Michel Crozier et Claude Lefort, s'est intéressé à toutes les relations affectives que l'individu entretient avec l'organisation. S'inspirant largement de la psychologie sociale contenue dans l'œuvre de Freud, telle qu'elle s'exprime dans Psychologie des foules et Analyse du moi, Totem et Tabou et Malaise dans la civilisation, (1971), ce groupe qui a constitué un véritable foyer intellectuel de 1959 à 1968 et a influencé de nombreux chercheurs en psycho-sociologie des organisations tente de rendre compte des investissements affectifs "sans lesquels aucun groupe organisé ne parviendrait à se constituer" (Enriquez, 1983, p. 57). Mettant l'accent sur l'amour, l'affectivité dans les rapports que les individus entretiennent entre eux, les chercheurs appartenant à ce courant essaient de mettre en lumière une dimension largement ignorée par les courants dominants. C'est ainsi que Eugène Enriquez, un des chefs de file du 
courant, s'est attardé à faire une psychanalyse du lien social $(1972,1983,1987)$, que Max Pagès a tenté de rendre compte de la vie affective des groupes (1968), que d'autres comme M. Pagès, M. Bonnetti, V. de Gauléjac et D. Descendre ont tenté d'articuler une compréhension d'inspiration freudo-marxiste de la vie humaine dans les organisations (1979), qu'André Lévy, Jean Dubost, Guy Palmade ont cherché à rendre compte de l'intensité affective des relations professionnelles ${ }^{13}$, que $\mathrm{D}$. Anzieu a tenté d'éclaircir la dynamique inconsciente des groupes (1975), que Nicole Aubert, E. Enriquez et de Gauléjac et de nombreux autres se sont penchés sur le sexe du pouvoir (1985) ou encore que G.N. Fischer a mis en lumière les liens qui unissaient les personnes à leur espace organisationnel $(1980,1989)$.

En réaction aux premiers travaux de l'ARIP, un autre courant s'est constitué. Regroupé autour de G. Lapassade et $R$. Lourau ce courant tire son inspiration principalement de la psychanalyse, du marxisme et en particulier de la pensée de Castoriadis à qui il emprunte les termes instituant et institué (1975). Son objectif est non seulement de décomposer ce qui est observable mais aussi et surtout de révéler ce qui se cache derrière les comportements apparents. Il s'agit de mettre au jour l'institution et par là même mettre en lumière les contradictions sociales (1974) car "l'institution est l'inconscient politique de la société" (Lapassade, 1972, p. 77). Produit du refoulement des relations sociales et de leur reproduction, l'institution bloque l'expression, aliène les individus et empêche les changements. Au nom d'un certain marxisme, et de larges emprunts à la pensée de Castoriadis, l'analyse institutionnelle se présente comme une contre-sociologie des organisations où ce qui est établi, à savoir l'institué, est toujours contesté par l'instituant, qui est lui-même soumis à la volonté d'institutionnalisation de l'institué. C'est de cette dialectique instituant-institué que l'institution surgit (Lapassade, 1970; Lourau, 1970). Fondamentalement critiques, l'analyse institutionnelle et sa méthode d'intervention, la socio-analyse, sont en droite ligne les héritières des bouleversements sociaux de la fin des années soixante et du début des années soixante-dix, en particulier à travers l'influence qu'ont exercée les événements de mai 68 sur la psychologie sociale. Si la socio-analyse cherche à intervenir dans les organisations pour les transformer, elle ne précise pas pour autant le type d'organisation qui devrait en découler. La plupart de ses interventions ont souvent plongé les organisations analysées dans un désordre souvent indescriptible (Lapassade, 1971), ce qui a conduit beaucoup de gens à s'en détourner. Toutefois, certaines de leurs idées ont profondément marqué l'analyse psycho-sociologique des organisations de langue française (Petit, 1979).

Parallèlement ou plus ou moins en relation avec le premier courant, deux autres courants ont également vu le jour dans les dix dernières années: celui de la psychopathologie du travail animé par le psychanalyste français Christophe Dejours et le courant "psychanalyse et management", issu des Écoles de gestion.

13 Voir notamment, leurs articles dans la revue Connexions et l'ouvrage collectif de Paris IX Dauphine (1987). 
Aux confluents de la psychanalyse et des sciences sociales, la psychopathologie du travail cherche à comprendre et à interpréter le plaisir et la souffrance humaine dans les organisations (Dejours 1980). En montrant comment les modes d'organisation du travail entrent en contradiction avec l'appareil psychique de l'être humain, cette discipline en émergence vient à interroger les modes d'organisation, voire la gestion des entreprises (Dejours, 1988, 1990; J.-F. Chanlat, 1990). Appliquée à des univers de travail fort variés comme les centrales nucléaires, les chemins de fer, les industries chimiques, l'aviation militaire (Dejours, 1977, 1985, 1987, 1988), ou l'univers hospitalier (Carpentier-Roy, 1991), la psychopathologie du travail tente de réhabiliter la vie symbolique, souvent malmenée dans les ensembles organisés et jette un regard différent sur ce que les chercheurs anglophones appellent le stress au travail (J.-F. Chanlat, 1990).

Le courant "psychanalyse et management" quant à lui est issu des écoles de gestion. Ce sont des psychanalystes ou des professeurs ayant une culture psychanalytique qui, dans la mouvance des travaux analogues réalisés en langue anglaise ${ }^{14}$, s'intéressent à des phénomènes comme l'imaginaire, le leadership, la dynamique des groupes ou la stratégie d'entreprise. S'appuyant sur la psychanalyse, ils cherchent à interpréter les comportements des cadres de direction et à voir comment certains traits psychologiques peuvent affecter la dynamique des organisations. C'est ainsi que Laurent Lapierre dans une perspective kleinienne s'est penché sur le leadership $(1987,1988)$, que Thierry Pauchant influencé par la pensée de Kohut s'intéresse au narcissisme et à la gestion des crises (1990) et que Gilles Amado, spécialiste des groupes (1975), propose des articulations dialectiques entre les structures organisationnelles et les processus inter-humains (1986) ou les crises individuelles (1990) et construit une perspective transitionnelle du management, inspirée par les travaux du psychanalyste britannique Winnicott (1989).

La culture et l'affectivité ne sont toutefois pas les seuls phénomènes sur lesquels se sont penchés les chercheurs de langue française. D'autres courants témoignent également de la variété des contributions du champ francophone.

\section{Le courant actionnaliste}

Représenté principalement par les travaux d'Alain Touraine et de ses disciples, le courant actionnaliste est d'abord et avant tout un courant dont l'influence s'est exercé et s'exerce encore dans les pays latins (France, Amérique latine, Québec, etc.). Pour l'actionnalisme, le sujet de l'histoire, producteur de la société, ce sont les mouvements sociaux. Il se définit comme un courant post-marxiste qui "s'efforce de construire l'ensemble de l'organisation sociale et culturelle à partir de

14 Notamment les travaux de Zaleznick (1970), Zaleznick et Kets de Vries (1975), et ceux du Tavistock: Jaques (1955), Bion (1959), Menzies (1960). 
la connaissance du mode de production de la société par elle- même" (1969, p. 179). Partant d'un schéma général appelé système d'action, qui s'apparente quelque peu aux efferts de formulation parsoniens, l'organisation, loin d'être considérée comme un système de relations sociales organisé autour de certaines normes de communication et d'autorité, apparaît, comme écrit Touraine, dans la perspective actionnaliste, comme une médiation, qui peut être aussi un obstacle, entre les acteurs historiques, dirigeants aussi bien que salariés, et les valeurs de développement et de démocratie $(1965$, p. 183). L'organisation ne peut être assimilée à la société, car elle dépend des intérêts particuliers. Cette particularité fait naître une double dialectique. D'une part, une dialectique interne mettant aux prises les différents groupes de l'organisation et, d'autre part, une dialectique externe entre l'organisation et son environnement. Ni tout à fait dépendante, ni tout à fait autonome, l'organisation se situe dans un champ historique donné et dans un système de rapports de classe. L'organisation est donc traversée par les grands enjeux et les principaux conflits qui agitent la société qui l'environne ${ }^{15}$. En ce sens, la sociologie actionnaliste des organisations n'est pas dissociable d'une macro-sociologie (Touraine, 1973).

Dans le monde francophone, ce courant de recherche a débouché sur de nombreuses publications. Touraine et son équipe ont abordé tour à tour les mouvements étudiants (1968), le gauchisme (1978), les groupes écologistes (1980), le syndicalisme libre en Pologne (1982), le mouvement ouvrier (1984). $\mathrm{Au}$ Québec, où l'analyse actionnaliste a inspiré de nombreux chercheurs, on a vu apparaître des travaux sur une grève historique (David, 1977), le mouvement nationaliste (Gagnon, 1978), l'écologisme (Vaillancourt, 1981), la lutte antinucléaire (Babin, 1981) ou le mouvement social (Bélanger et Lévesque, 1987).

\section{Le courant systémique}

À l'instar des pays anglophones qui ont joué un rôle important dans le développement de l'idée de système, les pays de langue française ont également, au cours des années soixante-dix et quatre-vingt, vu émerger un courant systémique pour analyser l'organisation. C'est en s'appuyant sur les travaux réalisés dans les sciences biologiques et physiques qui ont mis en avant les concepts de système, d'auto-organisation, de complexité, qu'un certain nombre de travaux ont vu le jour ${ }^{16}$. C'est ainsi que certains chercheurs se sont penchés sur les théories de l'auto-organisation (Dumouchel et Dupuy, 1983) et sur leur application au domaine social (Castoriadis, 1983) ou organisationnel (Lemoigne et Carré, 1977; Morin, 1986). D'autres se sont intéressés à analyser les organisations en tant que

15 Voir en particulier le chapitre "l'entreprise : pouvoir, institution et organisation" dans A. Touraine, La société post-industrielle, Paris, Denoël-Gauthier, 1969, p. 189. 260.

16 Deux des revues qui rendent compte de ces travaux sont la revue lyonnaise Analyse de systèmes animée par J.-P. Algoud et la Revue internationale de systémique. 
systèmes (Bonis, 1975; Boisvert, 1980) ou à critiquer les théories de l'organisation d'un point de vue systémique (Lussato, 1972), ou encore à appliquer certaines notions de la systémique aux sciences de la gestion. C'est le cas notamment des travaux de Jacques Mélese sur les systèmes de gestion $(1968,1972)$, ceux de Nadeau et Landry (1986) sur les systèmes d'aide à la décision, des études de Lemoigne (1973), Landry (1975), Tardieu, Nanci et Pascot (1979), celles de Tardieu, Rochfeld et Colleti (1983) et de Landry, Pascot et Ridjanovic (1990) sur les systèmes d'informations organisationnels, des recherches de Lemoigne (1979), Bruyne (1981), Roy (1983) et Sfez $(1976,1989)$ sur la décision, et enfin celles de Landry $(1978,1983,1985,1987,1988)$ sur la formulation et la résolution de problèmes. Enfin, tout récemment, dans la foulée des recherches faites dans le domaine des sciences de la cognition, quelques chercheurs, notamment P. Cossette n'ont pas hésité à appliquer l'idée de cartographie cognitive à l'univers organisationnel $(1988,1988)$.

\section{Le courant socio-politique}

Dans le monde francophone, les organisations ont toujours été vues comme un champ éminemment politique. Depuis les premiers travaux de Crozier jusqu'à aujourd'hui, nombreux sont les chercheurs de langue française qui ont en effet accordé aux phénomènes politiques la première place. Si les tenants du courant de l'analyse stratégique ont plutôt tendance à faire des analyses de micro-sociologie politique (Dion, 1980), d'autres chercheurs ont essayé d'articuler les relations politiques internes aux rapports de pouvoir que l'on pouvait observer dans la société globale (Karpik, 1972a, 1972b; Jarniou, 1981). Les thèmes qui ont à cet égard suscité le plus d'intérêt sont la participation et la démocratie organisationnelle, les élites, les réseaux, les professions et les femmes.

Les expériences démocratiques ont été particulièrement bien étudiées dans les pays de langue française. Le goût pour le changement social et l'intérêt pour le pouvoir ont poussé de nombreux chercheurs à s'intéresser à ce que les AngloSaxons ont qualifié de démocratie industrielle. C'est ainsi que A. Meister s'est centré sur les expériences yougoslave et péruvienne (1982), que Y. Bourdet (1982) a développé une importante réflexion sur l'autogestion, que Mermoz a rendu compte des difficultés d'une expérience communautaire (1978), que Grand'Maison (1975), Boucher et Martel (1982) ont cherché à analyser le passage d'une entreprise privée québécoise à un système autogéré, que de nombreux autres, notamment $\mathrm{H}$. Desroche (1976) et Claude Vienney (1980) se sont penchés sur les coopératives et les expériences autogestionnaires ${ }^{17}$ ou A. Joyal et $\mathrm{H}$. Bhérer se sont intéressés aux entreprises alternatives (1987).

17 Sur ce sujet, un grand nombre de travaux ont été publiés dans les revues québécoises Possibles et Coopératives et développement et les revues françaises Autogestion et Archives internationales de sociologie de la coopération et du développement. 
D'autres ont analysé l'exercice démocratique dans de petites organisations (entreprises artisanales, cabinets professionnels, associations culturelles, entreprise alternatives etc.) (Sainsaulieu, Tixier et Marty, 1983) ou encore la tension entre l'association et l'entreprise au sein des coopératives (Lévesque, Desforges, Tremblay, 1979). Enfin certains se sont penchés plus particulièrement sur les phénomènes de la représentativité des usagers dans les institutions publiques (système de santé, services sociaux, organismes populaires, école, etc.) et à leur subordination aux catégories techno-bureaucratiques (Godbout, 1977, 1983; Divay, 1979, 1977).

Le gouvernement des entreprises publiques, privées ou coopératives, leur direction et leur choix ont constitué un autre sujet de recherche pour les chercheurs d'expression française. À cet égard, plusieurs travaux importants ont cherché à mieux cerner les élites dirigeantes. C'est ainsi que A. Sales a procédé à une véritable radioscopie des élites industrielles québécoises (1979), que J. Niosi a tenté de mieux cerner l'establishment canadien (1980), que P. Fournier a fait une étude sur le patronat québécois (1979), que A. Sales et N. Bélanger ont procédé à une recherche sur les gestionnaires des secteurs privé et public (1985), que Bauer et Cohen ont proposé, après avoir étudié plusieurs grands groupes industriels français, la notion de système dirigeant, le pouvoir de ce dernier s'appuyant sur une expertise légitime, contredisant ainsi la thèse de Galbraith sur la technostructure, et que Simard a suivi la longue marche des technocrates québécois (1979). Un certain nombre de chercheurs se sont intéressés par ailleurs aux élites professionnelles, notamment aux comptables (Séguin et Hamel, 1982), et aux ingénieurs québécois (Séguin et Hubert, 1983).

L'arrivée des femmes sur le marché du travail a également suscité de nombreuses recherches. Ces études se sont non seulement intéressées au fait féminin en soi (Sullerot, 1978) mais aussi aux processus d'articulation et de renforcement mutuel entre le rapport au travail des femmes et leur place au sein de l'institution familiale (Barrere-Maurisson, 1984; David, 1986; D. Gaucher, 1983; Vandelac et coll. 1985). À l'intérieur de ce cadre, plusieurs chercheurs ont porté leur attention sur les cadres féminins. En France, ces travaux ont été menés dans l'optique d'un processus de construction d'une identité sociale nouvelle. On a ainsi cherché principalement à explorer la constitution de la différenciation sexuelle en milieu organisationnel (Hupper-Laufer, 1982), à décrire comment les différentes figures de l'identité féminine déterminaient diverses manières d'exercer le pouvoir (Aubert, 1982) et à voir comment l'identité professionnelle des femmes cadres travaillant dans un même secteur industriel pouvait varier selon la culture des entreprises (Belle, 1990).

Au Québec, après avoir cherché à définir la situation des femmes cadres (HarelGiasson, 1981), de nombreuses recherches ont voulu rendre compte du vécu des cadres féminins, des difficultés auxquelles elles pouvaient faire face dans différents types d'organisation (Harel-Giasson et Robichaud, 1988; Simard, 1983; Baudoux et 
de la Durantaye, 1988) et des traces possibles que la présence de femmes cadres peut laisser au niveau de l'organisation (Harel-Giasson, 1990).

Enfin, d'autres chercheurs ont distingué le pouvoir des appareils de celui des réseaux (Lemieux, 1981) ou ont montré l'importance que joue le réseau dans les entreprises publiques, et dans la création d'entreprises privées (Arocena, PaulCavallier, Minguet, Richard, Mennaux, 1983; Arocena, 1986) ou encore dans la production des faits scientifiques (Callon, 1989). L'action d'une entreprise ou d'une organisation quelle qu'elle soit ne peut alors se comprendre sans renvoyer au tissu social dans lequel elle s'insère et aux relations socio-politiques qu'elle y a développées (Hamel, Houle et Sabourin, 1984; Bélanger et Fournier, 1987; Wieviorka et Trinh, 1989).

\section{Le courant "anthropologique"}

Le monde des organisations, et notamment celui de la gestion, a également vu surgir un courant anthropologique au sens étymologique ou encore maussien de ce terme. Ce courant cherche à comprendre les phénomènes humains observés dans les organisations à partir des données accumulées par "le total des sciences qui, comme l'écrivait Mauss au début de ce siècle, considèrent l'homme comme un être vivant, conscient et sociable" (1968, p. 285). Développée en France par Maurice Dufour, il y a maintenant une vingtaine d'années, reprise et poursuivie par Alain Chanlat à l'École des hautes études commerciales de Montréal et par d'autres chercheurs de cette institution (Chanlat, 1990), cette approche se veut fondamentalement pluridisciplinaire dans son intention et unitaire dans sa compréhension de l'être humain (Chanlat,1989; Chanlat et Dufour, 1985). Cette position épistémologique et théorique rejoint celle d'autres chercheurs contemporains qui tentent de rendre compte non seulement de la complexité humaine en général (Gusdorf, 1967; Morin, 1973, 1979; Serres, 1979; Bateson, 1989) mais aussi de celle qui apparaît dans les organisations (Morgan, 1986, 1989; Girin, 1982; Berry, 1986; Enriquez, 1983; Collectif, 1987).

Partant du constat que les sciences du comportement appliquées à la connaissance de la vie humaine dans les organisations restent encore trop réductionnistes dans leurs approches, trop utilitaires dans leurs intentions et largement marquées au sceau de l'ethnocentrisme occidental, voire américain, les travaux de ce courant tentent de montrer comment l'introduction de nouvelles disciplines (sciences du langage, linguistique, anthropologie, etc.) peut améliorer non seulement notre compréhension de l'univers organisationnel (Chanlat, 1984; Chanlat et Dufour, 1985), mais aussi édifier une anthropologie des organisations (Chanlat, 1990) et contribuer à la transformation des modes de gestion (Aktouf, 1989). 


\section{Le courant communicationnel}

La fin des années quatre-vingt a vu également émerger un courant qui, tout en s'appuyant sur des disciplines diverses et des paradigmes variés, s'est attaché à mieux cerner les communications au sein des organisations tant en France qu'au Québec $^{18}$.

Partant de l'idée que l'organisation est un lieu de communication (Taylor, 1988), ces travaux ont cherché à rendre compte des différentes facettes de ce phénomène. D'aucuns se sont appuyés sur les travaux de l'école de Palo Alto (Dionne et Ouellet, 1990); d'autres sur une conception d'auto-organisation (Laramée, 1990) ou encore sur une vision plus classique de la communication (Bartolik, 1990). Certains chercheurs se sont intéressés à la communication dans des organisations particulières (Beauchamp, 1989); d'autres aux enjeux stratégiques que les communications représentent dans tout l'ensemble organisé (Gendreau, 1989); d'autres, enfin, aux mécanismes discursifs (Giroux, 1989). Très présent au Québec, ce courant s'est bâti dans la mouvance de ce qui se faisait aux Etats-Unis où le champ de la communication organisationnelle est particulièrement développé (Jablin et coll., 1987). En France, malgré l'intérêt que portent nombre de chercheurs à ce sujet, notamment ceux qui sont regroupés autour de la revue bordelaise Communication et organisation, il semble que l'engouement tourne plus autour des phénomènes langagiers (Actes, 1989) qu'autour de la communication organisationnelle proprement dite.

Tout comme bien d'autres disciplines, la communication organisationnelle paraît être marquée par un éclatement des intérêts de recherche, une diversité des approches utilisées et des choix paradigmatiques. À travers le phénomène de la communication, elle cherche à comprendre l'organisation en faisant appel dans une large mesure aux éléments déjà existants. Par ailleurs, cette insistance sur un aspect particulier de la vie organisationnelle n'est pas sans relation avec ce que l'on observe aujourd'hui: prolifération des nouvelles techniques de communication, rôle et importance de la communication d'entreprise, intérêt pour la culture d'entreprise et les phénomènes symboliques, omniprésence des médias, etc. En s'attachant à comprendre la communication dans les organisations, ce courant ne fait que traduire une réalité de plus en plus évidente dans nos sociétés: son caractère stratégique (Breton et Proulx, 1989).

Le courant épistémologique

Enfin à l'instar d'autres disciplines, la multiplication des travaux qui se veulent scientifiques dans les sciences de l'organisation a amené tout récemment la création

18 Voir le numéro spécial sur "la communication organisationnelle" de la revue Communication et Information, vol. 11, no 1, 1990. 
d'un noyau de chercheurs francophones qui s'intéressent à la production des connaissances dans ce domaine (Audet et Malouin, 1986). Au Québec, inspiré par la théorie de la structuration de A. Giddens et les travaux en histoire des sciences, Michel Audet a été le premier à préciser le champ et les contours de cette démarche (1986).

Richard Déry, dans la foulée des travaux de Michel Audet et influencé par le constructivisme de J. Piaget, a procédé aux premières grandes études empiriques $(1989,1990 \mathrm{a}, 1990 \mathrm{~b}, 1992)$. Cet intérêt pour la réflexion épistémologique s'est également fait sentir dernièrement en France où plusieurs chercheurs s'interrogent sur les fondements de leur discipline (Martinet, 1990).

\section{L'analyse des organisations de langue française: un champ original}

À la lumière de ce qui précède, l'analyse des organisations de langue française apparait bel et bien comme un champ non seulement fort diversifié mais aussi original. Dans cette troisième et dernière partie, nous allons tenter d'avancer quelques hypothèses concernant l'originalité du champ francophone par rapport au champ anglo-saxon, notamment américain, et ce à un triple niveau: l'objet, la méthodologie et le champ lui-même.

\section{Une triple insistance au niveau de l'objet}

Lorsqu'on parcourt les travaux de langue française en ayant en tête ceux de langue anglaise, et plus particulièrement la majorité des travaux américains on ne peut s'empêcher de remarquer les différences qui les séparent. Parmi toutes les distinctions que l'on peut faire, trois apparaissent particulièrement importantes: tout d'abord, la centralité accordée aux processus politiques, ensuite, l'importance donnée au sens et aux significations des actions humaines et enfin le rôle que jouent les aspects macro-sociaux et l'histoire dans l'interprétation des phénomènes observés.

Quel que soit le courant auquel le chercheur francophone appartient, il est rare en effet qu'il néglige la dynamique politique. Des premiers travaux de Crozier et de son équipe jusqu'aux toutes dernières études, nombreuses sont les recherches organisationnelles qui accordent volontiers une place prépondérante aux relations de pouvoir, aux conflits et au processus de légitimation.

L'influence historique du marxisme dans les sciences sociales, la longue tradition d'analyse critique qui parcourt la pensée française de Proudhon à Foucault en passant par Gurvitch et Sartre pour ne nommer que ceux-là, le caractère conflictuel des relations sociales, notamment en France, le désir d'émancipation sociale entretenu par de nombreux intellectuels, le goût prononcé pour la théorie et 
la compréhension du pourquoi ont poussé et poussent encore les chercheurs francophones et plus particulièrement les chercheurs français dans leur majorité à voir l'organisation comme un champ politique où s'exercent différents modes de domination (Tixier, 1988).

En s'appuyant sur une vision plutôt radicale du pouvoir (Lukes, 1974; Clegg, 1989), un grand nombre de travaux se distinguent nettement de la vision fonctionnaliste dominante mise en avant jusqu'à présent par la plupart des travaux nord-américains et se rapprochent davantage de certaines perspectives développées par certains Britanniques (Clegg et Dunkerley, 1980; Beynon, 1973; Allen, 1975; Clegg, 1989, 1990) et Américains (Braverman, 1974; Marglin, 1973; Burawoy, 1979).

C'est ce qui explique en partie le peu d'intérêt que les travaux anglophones d'inspiration structuro-fonctionnaliste ont jusqu'ici suscité dans ce domaine (Chanlat et Séguin, 1987; Séguin et Chanlat, 1988). S'appuyant sur une image plus consensuelle et fonctionnelle de l'organisation, les chercheurs anglophones ont souvent présenté dans leur majorité des analyses qui ne tenaient pas assez compte, pour les chercheurs de langue française, des rapports sociaux plus globaux et de leur historicité ${ }^{19}$. Ce faisant, ils ne pouvaient pas provoquer beaucoup d'intérêt chez leurs homologues de langue française. Seuls les travaux plus critiques étaient en mesure de le faire ${ }^{20}$.

Les chercheurs de langue française ne se sont pas contentés de focaliser sur les phénomènes de pouvoir. Ils ont également accordé beaucoup d'attention à la question du sens caché ou non des actions humaines. En puisant davantage dans les sciences du langage, la psychanalyse, l'anthropologie, la sociologie et la philosophie, ils n'ont pas hésité à donner à la symbolique et à l'imaginaire, notamment social pour reprendre une expression de Castoriadis (1975), une place que de nombreux homologues anglophones, appartenant en particulier au courant fonctionnaliste, rejetaient dans l'ombre. L'influence de la phénoménologie, notamment via la philosophie existentialiste de Sartre n'a pas été sans exercer un rôle important dans cette vision de la réalité organisationnelle. Ce phénomène est particulièrement observable dans les études sur les régulations culturelles. Contrairement à de nombreuses analyses anglo-saxonnes qui ont adhéré à une vision harmonieuse et instrumentale de la culture organisationnelle (Deal et

19 À cet égard, la redécouverte ou l'application de concepts marxistes que le champ de l'analyse des organisations a connu dans les pays de langue anglaise, à partir du milieu des années soixante-dix, ne pouvait apparaître comme une nouveauté pour les chercheurs de langue française, puisque ce courant de pensée avait toujours constitué pour eux un de leurs cadres intellectuels de référence privilégiés (Chanlat, 1989).

20 En dépit des objections émises parfois à leur endroit, les travaux critiques de langue anglaise ont toujours bénéficié d'un intérêt soutenu de la part des chercheurs francophones, ont été traduits souvent rapidement et ont été fort présents dans les débats. C'est le cas notamment des travaux de Braverman (1974) et de Marglin (1973). 
Kennedy, 1982), les chercheurs de langue française, nourris de pensée critique (Marx, Sartre, Foucault, Castoriadis, etc.) ont peu ou pas du tout partagé ce genre d'analyse. Bien au contraire, ils ont souvent vu dans cette montée de la notion de culture d'entreprise, une technique de manipulation symbolique.

En général, largement réfractaires à une vision behavioriste, les chercheurs de langue française sont demeurés très méfiants par rapport à toute idée qui s'apparente à une version plus ou moins sophistiquée du vieux schéma stimulus-réponse. En donnant de l'importance à des phénomènes qui relèvent avant tout de la subjectivité humaine, de l'inconscient ou de la symbolique, ils demeurent majoritairement critiques par rapport à toute conception qui ne tente pas d'aller au-delà des phénomènes observables. C'est ce qui explique par exemple que la critique de David Silverman, inspirée de Berger et Luckman, ait été généralement bien reçue dans les pays de langue française et constitue une des rares auvres de langue anglaise traduites rapidement en français (1973), que l'œuvre de Goffmann y jouisse aujourd'hui d'une large audience et que Garfinkel suscite beaucoup d'intérêt à l'heure actuelle en France (Coulon, 1987). Enfin, ils le sont d'autant plus que, d'une part, les travaux de Michel Foucault en insistant justement sur l'articulation des rapports symboliques et des rapports de pouvoir ont constitué dans les vingt demières années, pour nombre d'entre eux, un pôle de référence intellectuel notable (Foucault, 1975; Reitter et Ramanantsoa, 1985) et que, d'autre part, la psychanalyse a toujours bénéficié d'un certain intérêt auprès de plusieurs d'entre eux (Bernoux, 1985).

Si, jusqu'à présent, les chercheurs francophones semblent avoir porté dans l'ensemble plus d'attention aux phénomènes politiques, à la domination et aux éléments symboliques que leurs homologues américains appartenant au courant dominant, ils paraissent également faire plus souvent appel dans leurs analyses organisationnelles à des facteurs macro-sociaux. À l'exception de quelques travaux qui se centrent uniquement sur des phẻnomènes internes, nombreux sont en effet les travaux d'expression française qui ne dissocient jamais ce qui se passe à l'intérieur de l'organisation de l'environnement social plus global.

Une telle position s'explique par l'influence qu'ont exercée et exercent encore les théories macroscopiques du social. Les chercheurs de langue française, influencés par les grands systèmes théoriques principalement issus du marxisme et de l'actionnalisme, ont en effet du mal à dissocier l'organisation de son contexte socio-historique plus large. Contrairement à l'analyse traditionnelle de langue anglaise, telle qu'elle s'exprime surtout dans la majorité des travaux nordaméricains d'obédience fonctionnaliste et qui a développé la plupart du temps des conceptions a-historiques et a-sociologiques des organisations (Clegg et DunkerLey, 1977; Burrell et Morgan, 1979; Zey-Ferrell et Aiken, 1981; Clegg, 1990), les études menées par les chercheurs de langue française ont davantage fait appel aux rapports sociaux et à l'histoire démontrant par la même occasion combien les phénomènes organisationnels sont souvent interreliés, voire les échos de conflits sociaux, de débats idéologiques qui ont cours dans la société qui les environne. Cet 
intérêt pour la macro-analyse a été d'autant plus fort que la plupart de ces travaux ont été mus jusqu'à tout récemment par un désir de transformer à la fois les organisations et la société. Ce désir était particulièrement observable en France de la fin des années quarante à la fin des années soixante-dix, les uns prônant un modèle libéral (Crozier, 1970), la majorité des autres une transformation radicale. C'est en effet durant cette période, que l'on voit fleurir de nombreuses revues et publications qui tournent autour de l'autogestion, la transformation de l'organisation du travail et les expériences de contrôle ouvrier ${ }^{21}$. L'autodéveloppement social et organisationnel est alors vu comme le moyen de rompre avec l'institutionnalisation hétéronome et de libérer l'imaginaire (Castoriadis, 1975).

Aujourd'hui, les chercheurs francophones et plus particulèrement français sont devenus plus modestes. À l'image de ce qui s'est passé dans d'autres sociétés, le grand soir, la Révolution ne sont plus à l'agenda (Touraine, 1990). L'échec des solutions collectivistes, le déclin de l'État tutélaire, les difficultés des régimes tiersmondistes, la chute de l'orthodoxie marxiste, la redécouverte de l'acteur, du sujet, de la symbolique, la libération de la vie privée les ont poussés vers des positions plus nuancées ou encore, à l'instar de P. Bourdieu (1987) en France ou de A. Giddens (1987) en Angleterre, à dépasser les oppositions traditionnelles: individu-société, acteur-structure. Ce dépassement a eu des adeptes dans le domaine des organisations puisque Alain Eraly en Belgique (1988) et Hamid Bouchiky (1990) en France ont tenté tout dernièrement d'appliquer ces idées à l'analyse des structures organisationnelles.

L'originalité des travaux d'expression française semble donc reposer, au niveau de l'objet, sur cette triple insistance qui les parcourt, à savoir la centralité des phénomènes politiques, l'importance accordée à la question du sens et le rôle joué par les rapports socio-historiques. Cette sensibilité particulière devait amener les chercheurs francophones à avoir recours, comme nous allons le voir maintenant, à des méthodes davantage qualitatives.

Une méthodologie largement qualitative

Si le choix de l'objet détermine dans une large mesure le choix de la méthode (Devereux, 1980), les chercheurs de langue française, en s'attardant à étudier la dynamique humaine du point de vue des acteurs, de l'expérience et du vécu, ont pour un grand nombre d'entre eux fait appel à des méthodes qualitatives.

21 La revue Socialisme ou Barbarie, animée par Castoriadis, a poursuivi de 1949 à 1965 une réflexion originale sur la révolution dans le monde moderne, et a constitué un pôle intellectuel important dont l'influence se fait sentir encore aujourd'hui dans certains travaux. Voir également les ouvrages de Castoriadis portant sur la société bureaucratique (1973) et l'expérience du mouvement ouvrier (1974). 
Moins influencés par le modèle orthodoxe de la science que leurs collègues anglophones, notamment américains, ils n'ont jamais attribué aux recherches quantitatives le statut que ces derniers conferent à la mathématisation du social. Aux questionnaires fermés, à l'observation partielle et souvent élargie ils préfêrent nettement l'enquête de terrain, l'observation participante et l'analyse clinique (Aktouf, 1987). C'est la raison pour laquelle un grand nombre de recherches que nous avons présentées dans la partie précédente s'appuient davantage sur de telles méthodes. De façon générale, les chercheurs francophones privilëgient la connaissance en profondeur plutôt qu'en étendue. Étudier les dynamiques humaines telles qu'elles surgissent et les interpréter à la lumière de ce que les principaux acteurs font et disent, tels sont les objectifs poursuivis par la majorité des chercheurs de langue française. Plus souvent qu'autrement, cela les amène à pratiquer ce que les chercheurs anglophones ont qualifié de "grounded theory". Aux protocoles d'études quantitatives on préfère nettement des hypothèses générales de recherche. À la vérification d'hypothèses élaborées en laboratoire on substitue plus souvent qu'autrement l'interpellation quotidienne du terrain (Girin, 1982; Berry, 1986). Se laisser guider par les phénomènes qui surgissent de l'enquête et de l'analyse est largement préférable pour bon nombre d'entre eux à une multitude de travaux statistiques qui ne rendront pas toujours compte de l'expérience humaine.

Même si l'on peut trouver des études utilisant une méthodologie quantitative, ce parti pris méthodologique est largement partagé, plus peut-être encore en France qu'au Québec. Ce qui fait que les méthodes qualitatives ont bénéficié d'une légitimité souvent plus grande dans les pays de langue française que dans les pays de langue anglaise et notamment qu'aux États-Unis, surtout entre la fin des années soixante et le début des années quatre-vingt. C'est d'ailleurs un paradoxe. En effet, lorsqu'on jette un regard sur les travaux qui deviennent des classiques dans le champ de l'analyse des organisations (tant d'ailleurs en langue anglaise qu'en langue française), on ne peut s'empêcher de constater l'importance que les monographies, les analyses de cas approfondies ont eu et ont encore par rapport à la multitude des recherches statistiques existantes (Ballé, 1990). Cela prouve bel et bien la fécondité de ces méthodes. En s'appuyant dans leur majorité sur une solide tradition qualitative dans le domaine des sciences humaines, les chercheurs d'expression française, intéressés par la dynamique des organisations, ne pouvaient accorder à la quantification la place qu'elle semble occuper aux États-Unis depuis la fin des années soixante. Cela explique, là encore, le désintérêt que la plupart des études quantitatives effectuées outre-Atlantique ont connu dans les pays de langue française, en particulier en France, en revanche, la popularité de certaines enquêtes de terrain et analyses cliniques réalisées par des chercheurs anglophones qu'ils soient nord-américains ou britanniques ${ }^{22}$, ces dernières rejoignant beaucoup plus la sensibilité francophone, particulièrement française.

22 Parmi celles-ci, on peut retenir les études de Selznick (1949), Trist et Bamforth (1951), Gouldner (1954), Chandler (1962), Goffman (1968), Jaques (1972), Becker (1963) et plus récemment, celles de Mintzberg (1973) et de Pettigrew (1985) pour n'en nommer que quelques-unes. 
Différente dans une large mesure par ses intérêts de recherche et sa méthodologie, l'analyse des organisations de langue française semble constituer bel et bien un champ à part entière.

Un champ à part entière

Historiquement, il ne fait aucun doute pour un observateur averti que l'analyse des organisations formelles a émergé dans les pays anglophones et notamment aux États-Unis. Cet intérêt pour les ensembles organisés a débouché sur un champ scientifique largement dominé par les travaux nord-américains. Les chercheurs de langue anglaise ont dû, dans une large mesure, se situer par rapport aux débats engendrés par ces travaux. Le nombre de ses chercheurs, l'importance de ses revues et de ses congrès ont conféré avec le temps à l'Amérique du Nord une certaine hégémonie dans ce domaine.

Passage obligé de la reconnaissance académique, l'Amérique du Nord a imposé depuis bon nombre d'années, aux chercheurs d'autres provenances, ses débats, ses façons de penser, ses méthodes et bien sûr sa langue. Devant cette réalité, le champ francophone apparaît comme un de ceux qui, jusqu'ici peut-être, a le mieux résisté au rouleau compresseur anglo-américain. Si les travaux de Crozier et de bien d'autres chercheurs ont constitué des réponses à une certaine tradition angloaméricaine, il reste que l'univers linguistique, la tradition intellectuelle et les contextes socio-historiques, fort différents d'un univers à l'autre, ont maintenu une relative autonomie du champ francophone par rapport au champ anglo-saxon, notamment américain, ce phénomène étant particulièrement vrai pour la France et beaucoup moins pour les autres pays francophones, notamment pour le Québec.

Cette autonomie s'exprime au niveau du champ des organisations de différentes manières. Tout d'abord, par une organisation propre. Tout comme dans le champ anglo-saxon, le champ francophone possède ses revues, ses maisons d'édition, ses instituts, ses laboratoires de recherche, ses associations scientifiques, ses congrès et ses colloques. Jusqu'à il y a quelques années, il était donc possible de faire carrière dans ce champ sans avoir publié en anglais. À l'heure actuelle, cela est beaucoup moins le cas en France en raison des nouvelles politiques du CNRS et du rôle joué par les écoles de gestion en général qui encouragent tous deux les publications en langue anglaise, en particulier pour les jeunes chercheurs. Ensuite, cette organisation particulière véhicule des modes de pensée différents. Comme nous l'avons vu dans les pages précédentes, la recherche francophone, et plus particulièrement française, semble se caractériser par un certain nombre de particularités qui l'éloignent plus ou moins des travaux et des modes de pensée dominants aux États-Unis. Cette conscience d'être distinct est par ailleurs largement partagée. Les rejets du behaviorisme, du positivisme, et du fonctionnalisme constituent surtout en France un dénominateur commun. C'est une des raisons pour lesquelles peu de chercheurs ont publié jusqu'ici en langue anglaise. Compte tenu des positions théoriques et méthodologiques de certaines 
revues et des problèmes de traduction, on ne peut d'ailleurs guère s'en étonner ${ }^{23}$. De plus, l'attachement à publier dans sa langue est encore très fort chez les chercheurs de langue française. L'univers linguistique est en effet porteur d'une certaine conception du monde. On ne peut passer à une autre langue sans perdre en partie ce qui fait la particularité de la langue originelle et sans modifier ainsi la construction même de la réalité sociale. Enfin, cette autonomie, notamment dans le cas de la France, est renforcée par le caractère souvent hexagonal de la recherche française. À part de rares exceptions, les travaux de langue anglaise sont largement méconnus pour ne pas dire ignorés (Chanlat, 1989). La paresse linguistique, le manque de moyens des universités, notamment dans le domaine des bibliothèques, le dynamisme des sciences sociales françaises, le rôle central joué par l'intelligentsia parisienne dans les débats à la mode et le monde de l'édition (Hamon et Rotman, 1981), les distances intellectuelles en matière de pensée en sont les principales raisons. D'une certaine manière, cette méconnaissance est en quelque sorte le pendant de l'ignorance anglo-américaine pour les travaux de langue française. Nous sommes donc en présence d'un champ bel et bien distinct de celui de langue anglaise. Si son rayonnement est principalement latin pour des raisons d'affinités intellectuelles - on remarque en effet de nombreuses traductions des principaux ouvrages francophones en espagnol, italien, portugais et africain, pour des raisons historiques et linguistiques - on observe également une percée dans certains autres pays, notamment dans quelques pays de l'Est. Du côté des AngloSaxons, plus particulièrement des Britanniques, on a enfin remarqué au cours des dernières années un regain d'intérêt de la part de certains chercheurs pour des auteurs français comme Foucault, Callon, Latour ou Baudrillard (Clegg, 1989; Burrell, 1988). Du côté américain, l'ouverture d'une revue comme Organization Science à des problématiques extérieures au champ américain semble également amorcer de nouvelles relations dans ce domaine.

Au terme de cet article, le champ de l'analyse des organisations d'expression française apparaît sans aucun doute comme un champ relativement indépendant du champ anglo-saxon, notamment américain. Puisant à des traditions intellectuelles souvent différentes, utilisant des méthodes plutôt qualitatives, insistant sur des éléments fort distincts, et surgissant dans des sociétés singulières, les chercheurs d'expression française ont édifié des problématiques qui leur sont propres. Si les barrières linguistiques ont dans une large mesure empêché jusqu'ici qu'un dialogue s'instaure entre les deux univers, il ne faut pas non plus sous-estimer les différences d'ordre culturel et paradigmatique qui constituent, peut-être, des obstacles encore plus importants à l'établissement de ce dialogue. Toutefois, un

23 Cette attitude semble de plus en plus partagée par un nombre croissant de chercheurs anglo-saxons, notamment Britanniques (Burrell et Morgan, 1979; Clegg et Dunkerley, 1980; Hassard et Pym, 1990; Morgan, 1983, 1989; Reed, 1985). La création de Organization Studies avait déjà constitué en 1980 une réaction européenne à l'hégémonie américaine dans le domaine. L'éditorial du premier numéro de la revue américaine Organization Science (Daft et Lewin, 1990), fait aussi largement écho à ces préoccupations. 
tel dialogue peut exister, notamment entre les chercheurs qui, au-delà des différences linguistiques, partagent des points de vue intellectuels analogues. Dans certains cas, il existe déjà. Les colloques organisés sous l'égide de SCOS et d'EGOS en sont de vivants exemples.

Les chercheurs québécois en raison de leur double appartenance, à la fois nordaméricaine et française, sont peut-être en définitive les mieux placés pour influencer, voire renforcer les ponts déjà existants mais aussi pour briser en partie cette méconnaissance réciproque ${ }^{24}$. L'avenir nous dira si ces deux "solitudes" scientifiques pourront enfin instaurer un dialogue constructif et stimulant pour chacune des parties en empruntant ou non ce passage du Nord-Ouest auquel nous invitait, il y a quelque temps déjà, Michel Serres (1979).

\section{Jean-François CHANLAT professeur agrégé \\ École des Hautes Études commerciales}

Résumé

Depuis l'entre-deux-guerres où l'on voit apparaitre les premières études américaines sur la question, notamment celles d'Elton Mayo, l'intérêt pour les organisations n'a fait que croître dans la plupart des pays industrialisés. Dans les pays anglophones et plus particulièrement aux États-Unis, berceau des premiers travaux dans le domaine, l'analyse des organisations est devenue, dès le début des années soixante, un champ à part entière.

La croissance économique, la prolifération des organisations et leur développement, "la rationalisation du monde" ont en effet amené de plus en plus de chercheurs occidentaux à tenter de comprendre la dynamique sociale des ensembles organisés. Au cours des dernières années, cet intérêt a été renforcé par l'échec des solutions collectivistes, l'engouement pour l'entreprise et la gestion que connaît, de nos jours, l'Occident, et plus généralement par l'importance de penser le monde en termes d'organisation. Par rapport à cette tendance générale, que l'on peut aisément

24 Dans le domaine des organisations, il y a eu un effort fait dans ce sens. Les ouvrages de F. Séguin et J.-F. Chanlat (1988) et J.-F. Chanlat et F. Séguin (1987) ont permis aux lecteurs francophones d'avoir accès à plusieurs travaux classiques de langue anglaise. La collection "Sciences de l'administration", dirigée par Michel Audet aux Presses de l'Université Laval, a traduit G. Morgan (1989) et projette d'accueillir d'autres manuscrits de langue anglaise. Enfin, l'anthologie dirigée par R. Tessier et Y. Tellier (1990) en huit tomes a également contribué à cet effort. Dans le domaine de la pensée sociologique, ce n'est peut-être pas un hasard si le traducteur de A. Giddens (1987) soit Michel Audet lui-même. 
relever un peu partout dans les pays industrialisés, l'objet de cet article sera de montrer comment le champ francophone s'est développé et comment il se distingue encore aujourd'hui du champ anglo-saxon, notamment de l'analyse des organisations telle qu'elle se pratique majoritairement en Amérique du Nord.

Mots-clés: entreprise, organisation, gestion, études de l'organisation, champs d'études anglophones, champ d'études francophones.

\section{Summary}

The first organizational analyses were published in the United States during the interwar period, and the works of Elton Mayo assumed particular prominence. Since that time, interest in organizations has been growing steadily in industrial societies in general. Among the english-speaking countries, organizational analysis became a distinct discipline during the early $1960^{\circ}$ s, especially in its birthplace, the United States.

Economic growth, the proliferation of organizations and new organizational developments, and the "rationalization of the world" drove Western researchers towards an attempt to understand the social dynamics of formal organizations. This interest has been reinforced during recent years by the failure of collectivist regimes, an intensifying focus on the dynamics of enterprises and management in Western countries, and, more generally, the importance of viewing the world in organizational terms. With respect to this general tendency, which is evident in all industrial countries, the objective of this article is to demonstrate how organizational analysis has developed in francophone societies and how these orientations differ from those prevalent in english-speaking societies and especially in North America.

Key-words: enterprise, organization, management, organizational analysis, english-speaking, francophone.

\section{Resumen}

Desde el intervalo entre las dos guerras, en el que aparecen los primeros estudios americanos sobre el tema y principalmente los de Elton Mayo, el interés por las organizaciones no ha hecho sino crecer en la mayoría de los países industrializados. En los países anglófonos y más particularmente en los Estados Unidos, cuna de los primeros trabajos en el dominio, el análisis de las organizaciones se ha transformado, desde el comienzo de los años sesenta, en un verdadero campo de investigación. En efecto, el crecimiento económico, la 
proliferación de las organizaciones y su desarrollo, "la racionalización del mundo", han llevado cada vez más a los investigadores occidentales a intentar comprender la dinámica social de los conjuntos organizados. En el curso de los últimos años, este interés ha sido reforzado por el fracaso de las soluciones colectivistas, por el entusiasmo actual que conoce Occidente por la empresa y la gestión, y más generalmente, por la importancia de pensar el mundo en términos de organización. En relación con esta tendencia general, fácilmente detectable en los países industrializados, el objetivo de este artículo será mostrar de qué manera el campo francófono se ha desarrollado y cómo se distingue aún hoy del campo anglófono, particularmente del análisis de las organizaciones practicado mayoritariamente en América del norte.

Palabras claves: empresa, organización, gestión, estudios de la organización, campo de estudio anglófonos, campo de estudio francés. 


\section{Références bibliographiques}

ABRAVANEL, H. et al., La culture organisationnelle: aspects théoriques, pratiques et méthodologiques, Montréal, Gaëtan Morin, 1988.

ACTES DU COLLOQUE DE DOURDAN, La division du travail, Paris, Galilée, 1978.

ACTES DU COLLOQUE, Travail et pratiques langagières, Paris, CNRS, 1989.

AKERMAN, W. et SAINSAULIEU, R., L'étude sociologique du changement technique : pour une analyse stratégique, Paris, Publication du CERP, 1972.

AKTOUF, O., "Une vision interne des rapports de travail: le cas de deux brasseries", Le travail humain, 49 (3), septembre 1986, p. 238-248.

AKTOUF, O., Méthodologie des sciences sociales et approche qualitative des organisations, Montréal, Presses de l'Université du Québec, HEC Presses, 1987.

AKTOUF, O., Le management entre tradition et renouvellement, Montréal, Gaëtan Morin, 1989.

AKTOUF, O., "Symbolisme et culture d'entreprise : des abus conceptuels aux leçons de terrain", dans J.-F. CHANLAT (dir.), L'individu dans l'organisation : les dimensions oubliées, Québec, Paris, Presses de l'Université Laval, ESKA, 1990.

ALLEN, V. L., Social Analysis: A Marxist Critique and Alternative, Londres, Longman, 1975.

ALLAIRE Y. et FIRSIROTU, M.E., "Révolutions culturelles dans les grandes organisations: la gestion des stratégies radicales", dans H. ABRAVANEL, op cit., 1988, p. 197-226.

ALTER, N., La bureautique, Paris, Éditions Ouvrières, 1985.

ALTHABE, G., LÉGÉ, B. et SELIM, M., Urbanisme et réhabilitation symbolique, Ivry, Bologne, Amiens, Paris, Anthropos, 1984.

AMADO, G., "Cohésion organisationnelle et illusion collective", Actes du congrès international de psychologie du travail de langue française, Montréal, Les Presses de l'Université du Québec, 1987, p. 117-118.

AMADO, G. et GUITTET, A., La dynamique des communications dans les groupes, Paris, Armand Colin, 1975. 
AMADO, G., "Psychoanalysis and Organization", Sigmund Freud House Bulletin, vol. 4 , no 2,1980 , p. $17-20$.

AMADO, G., FAUCHEUX, C. et LAURENT, A., dans J.-F. CHANLAT (dir.), L'individu dans l'organisation: les dimensions oubliées, Québec, Paris, Les Presses de l'Université Laval, Éditions ESKA, 1990.

AMADO, G., "Psychopathologie du couple de direction", dans N. AUBERT, E. ENRIQUEZ, V. de GAULÉJAC, (dir.), Le sexe du pouvoir, Éditions de l'Épi, 1986 , p. $337-354$.

AMADO, G., "A Transitional Design for Management Learning", dans L. KLEIN (dir.), Working with Organizations, Londres, 1989.

AMADO, G., "Identité psychique, crise et organisation pour une théorie de la résonance", Psychologie Clinique, no 3, 1989, p. 103-115.

AMADO, G., "Organizational Behavior and Cultural Context: the Brazilian 'Jeitinho'", Congrès Mondial de Sociologie, Madrid, 9-13 juillet 1990.

ANZIEU, D., Le groupe et l'inconscient. L'imaginaire groupal, Paris, Dunod, 1975.

ARCHIER, G. et SERIEYX H., L'entreprise du 3ème type, Paris, Seuil, 1984.

ANTHROPOLOGIE ET SOCIÉTÉS, "Travail, industries et classes ouvrières", 10, $1,1986$.

AROCENA, J., Le développement par l'initiative locale, Paris, L'Harmattan, 1986.

AUBERT, N., Le pouvoir usurpé, Paris, Laffont, 1982.

AUBERT, N. ENRIQUEZ, E. et de GAULEJAC, V., Le sexe du pouvoir, Paris, Épi, 1986.

AUDET M. et J.-C. MALOUIN (dir.), La production des connaissances scientifiques de l'administration, Québec, Presses de l'Université Laval, 1986.

AUDET, M., "Le procès des connaissances de l'administration", dans M. AUDET et J.-C. MALOUIN (dir.), La production des connaissances scientifiques de l'administration, Québec, Presses de l'Université Laval, 1986, p. 23-56.

AUDET, M. LANDRY, M. et DÉRY, R., "Science et résolution de problèmes: liens, difficultés et voies de dépassement dans le champ des sciences de 
l'administration", Philosophie des sciences sociales - Philosophy of Social Sciences, 76, 1986b.

BABIN, R.,"La lutte anti-nucléaire au Canada", Sociologie et Sociétés, vol. XII, no 1,1981, p. 131-146.

BALLÉ, C. et PEAUCELLE, J.-L., Le pouvoir informatique dans l'entreprise, Paris, Éditions d'organisation, 1972.

BALLÉ, C., Sociologie des organisations, Paris, P.U.F, Que sais-je?, no 2497, 1990.

BAREL, Y., Le paradoxe et le système, Grenoble, Presses Universitaires de Grenoble, 1979.

BARRÈRE-MAURISSON, M.A. (dir.), Le sexe du travail: structures familiales et systèmes productifs, Grenoble, Presses Universitaires de Grenoble, 1986.

BARTOLI, A., Communication et organisation, Paris, Éditions d'organisation, 1990.

BATESON, G., Mind and Nature, New York, Bantam Books, 1979.

BAUER, M. et COHEN, E., Qui gouverne les groupes industriels?, Paris, Seuil, 1981.

BEAUCHAMP, M., La communication et les organisations coopératives, Montréal, Gaëtan Morin, 1989.

BECKER, H., Outsiders: Studies in the Sociology of Deviance, New York, The Free Press, 1963.

BÉLANGER, Y. et FOURNIER P., L'entreprise québécoise, développement historique et dynamique contemporaine, Montréal, HMH Hurtubise, 1987.

BÉLANGER, P. R. et LÉVESQUE B., "Le mouvement social au Québec: continuité et rupture (1960-1985)", dans P. R. BÉLANGER, B. LÉVESQUE, R. MATHIEU et F. MIDY, Animation et culture en mouvement. Fin ou début d'une époque?, Québec, Presses de l'Université du Québec, 1987, p. 253-266.

BELLE, F., "Les femmes cadres. Quelles différences dans la différence?", dans J.F. Chanlat, L'individu dans l'organisation : les dimensions oubliées, Québec, Paris, Presses de l'Université Laval, Éditions ESKA, 1990.

BERNOUX, P., Sociologie des organisations, Paris, Seuil, 1985. 
BERNOUX, P., MOTTE, D., SAGLIO, J., Trois ateliers d'Os, Paris, Éditions Ouvrières, 1973.

BERRY, M., Une technologie invisible? L'impact des instruments de gestion sur l'évolution des systèmes humains, Paris, CRG, 1983.

BERRY, M., "Logique de la connaissance et logique de l'action", dans M. AUDET et J.-C. MALOUIN, La production des connaissances scientifiques de l'administration, Québec, Presses de l'Université Laval, 1986, p. 181-231.

BERTAUX, D. et BERTAUX-WIAME, I., "Artisanal Bakery in France: How it lives and why it survives", dans F. Bechofer et B. Elliot (dir.), The Petite Bourgeoisie: Comparative Studies of the Uneasy Stratum, Londres, MacMillan, 1981, p. 121-154.

BEYNON, H., Working for Ford, Londres, Allen Lane, 1973.

BHÉRER, H., Le management soviétique, Paris, Fondation nationale des sciences politiques, 1982.

BION, W., Experience in Groups, New York, Basic Books, 1959.

BLAU, P. et SCOTT, W.R., Formal Organization, San Francisco, Chandler, 1962.

BOISVERT, M., L'approche socio-technique, Montréal, Agence d'Arc, 1980.

BONIS, J., Le système humain des organisations, Paris, Hommes et Techniques, 1975.

BOSCHE, M., "Corporate culture : la culture sans histoire", Revue française de gestion, nos 47-48, septembre-octobre, 1989, p. 29-39.

BOUCHARD, S., "Être truckeur" (routier)", dans A. CHANLAT et M. DUFOUR (dir.), La rupture entre l'entreprise et les hommes, le point de vue des sciences de la vie, Montréal, Paris, Québec/Amérique, Éditions d'organisation, 1985.

BOUCHER, P.-A et MARTEL, J.-L., Tricofil tel que vécu, Montréal, Les Presses HEC, Éditions CIRIEC, 1982.

BOUCHIKI, H., Structuration des organisations, Paris, Economica, 1990.

BOUDON, R., Effets pervers et ordre social, Paris, PUF, 1977.

BOURDET, Y. et GUILLERM, A., Les clefs pour l'autogestion, Paris, Seghers, 1975. 
BOURDIEU, P., Le sens pratique, Paris, Minuit, 1987.

BOUVIER, P., Technologie, transports, travail, Paris, Méridiens-Klincksieck, 1985.

BRAVERMAN, H., Labor and Monopoly Capital, New York, Monthly Review Press, 1974.

BRETON, P. et PROULX, S., L'explosion de la communication. La naissance d'une nouvelle idéologie, Paris, La Découverte, 1989.

BRUYNE, P. de, Modèles de décisions. Les rationalistes de l'action, Louvain, Centre d'études praxéologiques, 1981.

BURAWOY, M., Manufacturing Consent: Changes in the Labor Process under Monopoly Capitalism, Chicago, University of Chicago Press, 1979.

BURRELl, G. et MORGAN, G., Sociological Paradigms \& Organizational Analysis, Londres, Heineman Educational Book, 1979.

BURRELL, G., "Modernism, Post-modernism and Organizational Analysis: an Introduction", Organization Studies, 9/1, 1988, p. 91-112.

BURRELL, G., "Modernism, Post-modernism and Organizational Analysis: the Contribution of Michel Foucault", Organization Studies, 9, 2, 1988, p. 221-236.

CAILLIES, A., France - Japon - Confrontation culturelle dans les entreprises mixtes, Paris, Méridiens-Klincksieck, 1986.

CALLON, M. (dir.), La science et ses réseaux. Genèse et circulation des faits scientifiques, Paris, La Découverte, 1989.

CARPENTIER-ROY, M. C., Corps et âme. Psychopathologie du travail infirmier, Montréal, Liber, 1991.

CASTORIADIS, C., La société bureaucratique, Paris, UGE, 10/18, 1973.

CASTORIADIS, C., L'expérience du mouvement ouvrier, prolétariat et organisation, Paris, UGE, 10/18, 1974

CASTORIADIS, C., L'institution imaginaire de la société, Paris, Seuil, 1975.

CHANDLER, J., Strategy \& Structure, Cambridge, MIT Press, 1962.

CHANLAT, A., BOLDUC, A. et LAROUCHE, D., Gestion et culture d'entreprise, le cheminement d'Hydro-Québec, Montréal, Québec/Amérique, 1984. 
CHANLAT, A. et DUFOUR, M., La rupture entre l'entreprise et les hommes, le point de vue des sciences de la vie, Montréal, Paris, Québec/Amérique, Éditions d'organisation, 1985.

CHANLAT, J.-F. et SÉGUIN, F., L'analyse des organisations, tome II: Les composantes de l'organisation, Montréal, Gaëtan Morin, 1987.

CHANLAT, J.-F., "L'analyse sociologique des organisations; un regard sur la production anglo-saxonne contemporaine (1970-1988)", Sociologie du travail, no 3,1989 , p. 381-399.

CHANLAT, J.-F. (dir.), L'individu dans l'organisation: les dimensions oubliées, Québec,Paris, Les Presses de l'Université Laval, Éditions ESKA, 1990.

CHANLAT, J.-F., "Stress, psychopathologie du travail et gestion", dans J.-F. CHANLAT (dir.), L'individu dans l'organisation: les dimensions oubliées, Québec, Paris, Les Presses de l'Université Laval, Éditions ESKA, 1990, p. 709-722.

CLEGG, S. et DUNKERLEY, D., Organization, Class and Control, Londres, Routledge \& Kegan Paul, 1980.

CLEGG, S., Frameworks of Power, Londres, Sage, 1989.

CLEGG, S. (dir.), Organization Theory and Class Analysis, Berlin, de Gruyter, 1990.

COLLECTIF Sciences humaines, Paris IX, Dauphine, Organisation et management en gestion, Paris, L'Harmattan, 1987.

COULON, A., L'ethnométhodologie, Paris, PUF, Que sais-je?, 1987.

CROZIER, M., Le phénomène bureaucratique, Paris, Seuil, 1962.

CROZIER, M., La société bloquée, Paris, Seuil, 1970.

CROZIER, M. et THOENIG, J.-C., "The Regulation of Complex Organized Systems", Administrative Science Quarterly, vol. 21, no 4, décembre 1976, p. 547-570.

CROZIER, M. et FRIEDBERG, E., L'acteur et le système, Paris, Seuil, 1977.

CROZIER, M. et al., Où va l'administration française?, Paris, Éditions d'organisation, 1979.

CROZIER, M., Le mal américain, Paris, Seuil, 1980. 
CROZIER, M., État moderne. État modeste, Paris, Fayard, 1987.

DAFT, R.L. et LEWIN, A.Y. "Can Organization Studies Begun To Break out of the Normal Science Start Jacket? an Editorial Essay", Organization Science, vol. 1, no 1, p. 1-9, 1990.

DAVID, H., Femmes et emplois. Le défi de l'égalité, Montréal, Presses de I'Université du Québec, 1986.

DEAL, T.E. et KENNEDY, A. A., Corporate cultures: The rites and rituals of corporate life, Reading (MA), Addison-Wesley, 1982.

DEJOURS, C., VEIL, C. et WISNER, A., Psychopathologie du travail, Paris, Entreprise moderne d'édition, 1985.

DEJOURS, C. (dir.), Plaisir et souffrance dans le travail, Paris, CNRS, tome I, 1987.

DEJOURS, C. (dir.), Plaisir et souffrance dans le travail, Paris, CNRS, tome II, 1988.

DEJOURS, C., "Plaisir et souffrance dans les organisations", dans J. F. CHANLAT (dir.), 1990.

DENIS-GRENIER, H., Pouvoir et centralisation: une organisation québécoise, thèse de doctorat, Paris, 1974.

DENIS, H., Technologie et Société. Essai d'analyse systémique, Montréal, Éditions de l'École polytechnique, 1987.

DESFORGES, J.-G., LÉVESQUE, D. et TREMBLAY, B., "Dynamique de la coopérative: Association et entreprise", Gestion, avril, 1979, p. 39-48.

DESMAREZ, P., La sociologie industrielle aux États-Unis, Paris, Armand Colin, 1986.

DÉRY, R., La structuration discursive de la problématique de la décision dans la revue Administrative Science Quaterly: une contribution à l'épistémologie des sciences de l'organisation, thèse de doctorat présentée à la faculté des sciences de l'administration, Québec, Université Laval, 1989.

DÉRY, R., La structuration du champ de la stratégie, rapport de recherche, Montréal, École des hautes études commerciales, 1990a.

DÉRY, R., La structuration du champ de la décision, rapport de recherche, Montréal, École des hautes études commerciales, $1990 \mathrm{~b}$. 
DÉRY, R., "Enjeux et controverses épistémologiques dans le champ des sciences de l'administration", Revue canadienne des sciences administratives, vol. 9, no 1, 1992, p.1-2.

DESROCHES, H., Le projet coopératif, Paris, Éditions Ouvrières, 1976.

DEVEREUX, G., De l'angoisse à la méthode, Paris, Flammarion, 1980.

DION, S., "Pouvoir et conflit dans l'organisation: grandeur et limites du modèle de Michel Crozier", Revue canadienne des sciences politiques, 15, 1, 1980, p. 85102.

DION, S., La politisation des mairies, Paris, Economica, 1987.

DIONNE, P. et OUELLET, G., La communication interpersonnelle et organisationnelle: l'effet Palo Alto, Montréal, Gaëtan Morin, 1990.

D'IRIBARNE, P., "Vers une gestion culturelle des entreprises", Annales des mines. Gérer et comprendre, 4, septembre, 1986, p. 77-85.

D'IRIBARNE, P., La logique de l'honneur, Paris, Seuil, 1989.

DIVAY, G., Réforme institutionnelle et fourniture des biens collectifs locaux. Une approche socio-politique, thèse de doctorat, Québec, Faculté des sciences sociales, Université Laval, 1977.

DUPUIS, J.P., ""Anthropologie, culture et organisation" vers un modèle constructiviste", dans J.-F. Chanlat, L'individu dans l'organisation: les dimensions oubliées, Québec, Paris, Presses de l'Université Laval, ESKA, 1990.

DUPUY, F. et THOENIG, J.-C., La loi du marché, Paris, L'Harmattan, 1986.

DUMOUCHEL, L.-P. et DUPUY, J.-P. (dir.), L'auto-organisation. De la physique à la politique, Paris, Seuil, 1983.

DUPUY, F. et THOENIG, J.-C., L'administration en miettes, Paris, Fayard, 1985.

ENRIQUEZ, E., "Imaginaire social, refoulement et répression dans les organisations", Connexions psychologiques sciences humaines, 3, 1972, p. 65-93.

ENRIQUEZ, E., De la horde à l'État, Paris, Gallimard, 1983. 
ENRIQUEZ, E., "Personnalité et organisation" dans Collectif sciences humaines, Paris IX, Dauphine, Organisation et management en question, Paris, L'Harmattan, 1987, p. 11-28.

ERALY, A., La structuration de l'entreprise, la rationalité en action, Bruxelles, Éditions de l'Université de Bruxelles, 1988.

ETCHEGOYEN, A., Les entreprises ont-elles une âme?, Paris, François Bourin, 1990.

ETZIONI, A., Comparative Analysis of Complex Organizations, New York, Free Press, 1961.

FISCHER, G. N., Espace industriel et liberté, Paris, PUF, 1980.

FISCHER, N., Psychologie des espaces de travail, Paris, Armand Colin, 1989.

FOUCAULT, M., Surveiller et punir, Paris, Gallimard, 1975.

FOURNIER, M., TRÉPANIER, M. et GIRARD, S., "La sociologie dans tous ses états", Recherches sociographiques, XXVI, 3, 1985, p. 417-443.

FOURNIER, P., Le patronat québécois au pouvoir: 1970-1976, Montréal, HMH, 1979.

FREUD, S., Totem et Tabou, Paris, Payot, 1947.

FREUD, S., Malaise dans la civilisation, Paris. PUF, 1971.

FREUD, S., Essais de psychanalyse, Paris, Payot, 1981.

FRIEDBERG, E., L'analyse sociologique des organisations, Toulouse, Privat, 1971.

FRIEDBERG, E. et MUSSELIN, C., En quête d'université. Étude comparée des universités en France et en RFA, Paris, L'Harmattan, 1989.

FRIEDMANN, G., Problèmes du machinisme industriel, Paris, Gallimard, 1947a.

FRIEDMANN, G., Où va le travail humain, Paris, Gallimard, 1947b.

FRIEDMANN, G., Le travail en miettes, Paris, Gallimard, 1954.

FRIEDMANN, G., Sept études sur l'homme et la technique, Paris, Denoël Gonthier, 1966. 
FRIEDMANN, G. et NAVILLE, P., Traité de sociologie du travail, Paris, Armand Colin, 1961.

GAGNON, G., "Sociologie, mouvements sociaux, conduites de rupture : le cas québécois", Sociologie et Sociétés, vol X, no 2, 1978, p. 103-122.

GENDREAU, S., Communication organisationnelle : enjeu stratégique, Montréal, Département de communications, Université de Montréal, 1989.

GIDDENS, A., La constitution de la société, Paris, PUF, 1987.

GIRIN, J., "Un paradigme pour la recherche en gestion?", Économies et sociétés, série Sciences de gestion, no 2, octobre-décembre, 1981, p. 10-12.

GIRIN, J., "Langage en actes et organisations", Économies et sociétés, série Sciences de gestion, no 3, décembre 1982, p. 1559-1591.

GIRIN, J., "Problèmes du langage dans les organisations", dans J.-F. CHANLAT (dir.), L'individu dans l'organisation: les dimensions oubliées, Québec, Paris, Les Presses de l'Université Laval, Éditions ESKA, 1990., 1990.

GIROUX, N., "Mécanismes discursifs du retournement stratégique", Actes $d u$ colloque Travail et pratiques langagières, document ronéotypé, Paris, 1989.

GODBOUT, J., La participation contre la démocratie, Montréal, Saint-Martin, 1983.

GOFFMAN, E., Asylum, New York, Doubleday, 1961.

GORZ, A., Métamorphoses du travail, quête du sens. Paris, Galilée, 1988.

GORZ, A. (dir.), Critique de la division du travail, Paris, Seuil, 1973.

GOULDNER, A., Patterns of Industrial Bureaucracy, New York, The Free Press, 1954.

GRAND'MAISON, J., Une tentative d'autogestion, Montréal, Presses de l'Université de Montréal, 1975.

GRÉMION, P., Le pouvoir périphérique, Paris, Seuil, 1976.

GRÉMION, C., Profession: décideur, Paris, Bordas, 1979.

GUSDORF, G., Introduction aux sciences humaines, Strasbourg, Faculté des lettres de Strasbourg, 1960. 
HAMEL, J., HOULE, G. et SABOURIN R., "Stratégies économiques et développement industriel: l'émergence de Forano", Recherches sociographiques, XXV, no 2, mai-août, 1984, p. 89-209.

HAMON, H. et ROTMAN, P., Les intellocrates, Paris, Ramsay, 1981.

HAREL-GIASSON, F., Perception et actualisation des facteurs de promotion chez les femmes cadres des grandes entreprises québécoises francophones du secteur privé, thèse de doctorat, Montréal, École des HEC, 1981.

HAREL-GIASSON, F., "Femmes gestionnaires-l'actrice et l'organisation", dans J.-F. Chanlat, L'individu dans l'organisation: les dimensions oubliées, Québec, Paris, Presses de I'Université Laval, ESKA, 1990.

HAREL-GIASSON, F. et ROBICHAUD, J., Tout savoir sur les femmes cadres d'ici, Montréal, Presses HEC, 1988.

HASSARD, J. et PYM, D., The Theory and Philosophy of Organisation, Critical Issues and New Perspectives, Londres, Routledge, 1990.

HUPPERT-LAUFER, J., La féminité neutralisée? Les femmes cadres dans l'entreprise, Paris, Flammarion, 1982.

HININGS, R., "Defending Organization Theory: a British view from North America", Organization Studies, 1, 1988, p. 1-7.

JABLIN, F. M. et coll., Handbook of Organizational Communication: an Interdisciplinary Perspective, Beverly Hills, SAGE, 1987.

JACQUES, E., Intervention et changement dans l'entreprise, Paris, Dunod, 1978.

JARMOUS, H. et GRÉMION, P., L'ordinateur au pouvoir, Paris, Seuil, 1988.

JARNIOU, P., L'entreprise comme système politique, Paris, PUF, 1981.

JOYAL, A. et BHÉRER, H., Les entreprises alternatives, Montréal, Albert SaintMartin, 1987.

KAMDEM, E. , "Culture, temps et comportement au travail", Gestion, revue internationale de gestion, 11, 2, septembre, 1986, p. 36-43.

KAMDEM, E. , "Temps et travail en Afrique", dans J.-F. CHANLAT (dir.), J.-F. CHANLAT (dir.), L'individu dans l'organisation: les dimensions oubliées, Québec, Paris, Les Presses de l'Université Laval, Éditions ESKA, 1990. 1990. 
KARPIK, L., "Sociologie, économie politique et buts des organisations de production", Revue française de sociologie, XIII, 1972a, p. 299-320.

KARPIK, L., "Les politiques et les logiques d'action de la grande entreprise industrielle", Sociologie du travail, no 1, 1972b, p. 82-105.

KARPIK, L., "Organizations, Institutions and History", dans L. Karpik (dir.), Organization and Environment, Londres, Sage, 1978, p. 15-68.

KERGOAT, D., Bulledor: l'histoire d'une mobilisation ouvrière, Paris, Seuil, 1973.

LAMMERS, C., "Sociology of Organization Around the Globe. Similarities and Differences Between American, British, French, German and Dutch Brand", Organization Studies, 11/2, 1990, p. 179-205.

LANDRY, M., "Formation et résolution de problèmes en groupe dans un milieu organisationnel. Un schéma d'observation et d'analyse", Relations industrielles, $33,4,1978$, p. 591-609.

LANDRY, M., "Les problèmes organisationnels complexes et le défi de leur formation", Revue canadienne des sciences de l'administration, 5, 3, 1978, p. 3448.

LANDRY, M., PASCOT, P. et RIDJANOVIC, D., "Complexité et développement de systèmes d'information comme cycle de représentations", Technologies de l'information et société, 2(2), 1990.

LAPASSADE, G., Groupes, organisations et institutions, Paris, Gauthier-Villars, 1970.

LAPASSADE, G. et LOURAU, R., Clefs pour la sociologie, Paris, Seghers, 1974.

LAPASSADE, G., L'arpenteur, Paris, Épi, 1971.

LAPIERRE, L., "Imaginaire, leadership et gestion", Gestion, revue internationale de gestion, 12, 1, février 1987, p. 6-14.

LAPIERRE, L., "Puissance, leadership et gestion", Gestion, revue internationale de gestion, 13, 2 mai 1988, p. 39-49.

LAPIERRE, L., "Intériorité gestion et organisation", dans J.-F. CHANLAT (dir.), L'individu dans l'organisation: les dimensions oubliées, Québec, Paris, Les Presses de l'Université Laval, Éditions ESKA, 1990., 1990. 
LARAMÉE, A., "Une métaphore des systèmes auto-organisés comme cadre d'analyse et d'intervention en communication organisationnelle", Actes $d u 2^{e}$ colloque en communication organisationnelle, Montréal, Université de Montréal, 1990.

LARÇON, J.-P. et REITTER, R., Structure de pouvoir et identité d'entreprise, Paris, Nathan, 1979.

LATOUR, B. et WOOLGAR, S., La vie de laboratoire. La production des faits scientifiques, Paris, La Découverte, 1988.

LAUFER, R. et PARADEISE, C., Le prince bureaucrate, Paris, Flammarion, 1981.

LAURENT, A., "The Cultural Diversity of Western Conceptions of Management", International Studies of Management \& Organization, 13, 1-2, 1983, p. 75-96.

LEGENDRE, P., L'empire de la vérité, introduction aux espaces dogmatiques industriels, Paris, Fayard, 1983.

LEMAÎTRE, N., "La culture d'entreprise, facteur de performance", Gestion, février 1985, p. 19-25.

LEMAÎTRE, N., Le jeu de la décision. Pouvoirs, cultures et stratégie dans l'entreprise, Bruxelles, Éditions de l'Université de Bruxelles, 1986.

LEMOIGNE, J.-L., Le système d'information dans les organisations, Paris, PUF, 1973.

LEMOIGNE, J.-L., Les systèmes de décisions dans les organisations, Paris, PUF, 1974.

LEMOIGNE, J.-L., La théorie du système général. Théorie de la modélisation, Paris, PUF, 1977.

LEMOIGNE, J.-L. et CARRÉ, D., Auto-Organisation de l'entreprise. 50 propositions pour l'autogestion, Paris, Éditions d'organisation, 1977.

LINHART, R., L'établi, Paris, Minuit, 1978.

LIU, M., Approche socio-technique de l'organisation, Paris, Éditions d'organisation, 1983.

LOURAU, R., L'analyse institutionnelle, Paris, Minuit, 1970. 
LORSCH, J. (dir.), Handbook of Organizational Behavior, Englewood Cliffs, Prentice-Hall, 1987.

LUKES, S., Power: A Radical View, Londres, Macmillan, 1974.

LUSSATO, B., Introduction critique aux théories d'organisation, Paris, Dunod, 1972.

MARGLIN, S., "Origine et fonctions de la parcellisation des tâches", dans A. GORZ, Critique de la division du travail, Paris, Seuil, 1973.

MARTINET, A. C. (dir.), Épistémologies et sciences de gestion, Paris, Economica, 1990.

MATHEU, M., "Ethnographie et sciences des organisations", Enseignement et gestion, printemps 1983.

MATHEU, M., "Quelle familiarité distante. Quel regard poser sur la gestion dans notre société?", Annales des mines. Gérer et comprendre, mars 1986, p. 82-94.

MAURICE, M., SELLIER, F. et SYLVESTRE, J.-J., Politique industrielle en France et en Allemagne, Paris, PUF, 1982.

MEISTER, A., Où va l'autogestion Yougoslave?, Paris, Anthropos, 1971.

MÉLESE, J., La gestion par les systèmes, Paris, Hommes et techniques, 1968.

MÉLESE, J., L'analyse modulaire des systèmes de gestion, Paris, Hommes et Techniques, 1972.

MÉLESE, J., Approches systémiques des organisations. Vers l'entreprise à complexité humaine, Paris, Hommes et Techniques, 1979.

MERMOZ, M., L'autogestion c'est pas de la tarte, Paris, Seuil, 1978.

MENZIES, I," A Case Study in the Fonctionning of Social Systems as a Defense Against Anxiety", Human Relations, 13, 1960, p. 95-121.

MINGUET, G., La naissance de l'Anjou industriel, Paris, L'Harmattan, 1984.

MINTZBERG, H., The nature of managerial Work, New York, Harper \& Row, 1973.

MORGAN, G., Images de l'organisation, Québec, Paris, Presses de l'université Laval, Éditions ESKA, 1989. 
MORGAN, G. (dir.), Beyond Method, Beverly Hills, (CA), Sage, 1983.

MOSCOVICI, S., Modernisation des mines et changement technique, Paris, Publications du CERP-AFPH, 1963.

MORIN, E., Le paradigme perdu: la nature humaine, Paris, Seuil, 1973.

MORIN, E., "Complexité et organisation", dans M. AUDET et J. L. MALOUIN (dir.), La production des connaissances scientifiques de l'administration, Québec, Presses de l'Université Laval, 1986.

MOTHE-GAUTRAT, D., Pour une nouvelle culture d'entreprise, Paris, La Découverte, 1986.

MOTTEZ, B., La sociologie industrielle, Paris, PUF, Que sais-je?, 1970.

NADEAU, R. et LANDRY, M. (dir.), L'aide à la décision. Nature, instruments, perspectives d'avenir, Québec, Presses de I'Université Laval, 1986.

NIOSI, J., La bourgeoisie canadienne, Montréal, Boréal 1980.

NIZARD, G., "Identité et culture d'entreprise", Harvard l'expansion, 31, 1983, p. 90-106.

PADIOLEAU, J.-G., Le Monde et le Washington Post, Paris, PUF, 1985.

PAGES, M., La vie affective des groupes, Paris, Dunod, 1984.

PAGES, M., BONNETTI, V., DESCENDRE, D. et de GAUlEJAC, V., L'emprise de l'organisation, Paris, PUF, 1979.

PAUCHANT, Thierry, "La psychologie du self, l'expérience et la vie en entreprise une perspective kohutienne" dans J.-F. Chanlat, L'individu dans l'organisation: les dimensions oubliées, Québec,Paris, Les Presses de l'Université Laval, Éditions ESKA, 1990.

PAVÉ, F., L'illusion informaticienne, Paris, L'Harmattan, 1989.

PETIT, F., Introduction à la psychosociologie des organisations, Toulouse, Privat, 1979.

PETTIGREW, H., The Awakenning Giant: Continuity and Change in Imperial Chemical Industries, Oxford, Basil Blackwell, 1985. 
PIOTET, F., EXIGA, A. et SAINSAULIEU, R., Méthodes sociologiques d'analyse des conditions de travail, Paris, ANACT, 1981.

Possibles, "Faire l'autogestion", vol. 4, no 3-4, 1980.

RAIMBAULT, M. et SAUSSOIS, J.-M., Organiser le changement, Paris, Éditions d'organisation, 1983.

REITTER, R. et RAMANANTSOA, B., Pouvoir et politique : au-delà de la culture d'entrepise, Paris, McGraw-Hill, 1985.

REED, M., Redirections in Organizational Analysis, Londres, Tavistock Publications, 1985.

REYNAUD, J.-D., Les syndicats en France, Paris, Seuil, 1975.

REYNAUD, J.-D., Les règles du jeu l'action collective et la régulation sociale, Paris, Armand Colin, 1989.

RIBEIL, G., Les Cheminots, Paris, La Découverte, 1984.

RIVELINE, C., "Pour une ethnographie des organisations", Enseignement et gestion, printemps 1983 .

ROSANVALLON, P., La crise de l'État providence, Paris, Seuil, 1981.

ROSANVALLON, P., La question syndicale, Paris, Calmann-Lévy, 1988.

ROSE, M.J., French Industrial Studies, Farmborough, Hanto, Saxon House, 1977.

ROSE, M.J. (dir.), Industrial Sociology : Work in the French Tradition, Londres, Sage, 1987.

ROUSSEAU, N. (dir.), "Le culte de l'entreprise", Autrement, no 100, septembre 1988.

ROY, B. (dir.), La décision, ses disciples, ses acteurs, Lyon, Presses universitaires de Lyon, 1983.

SAINSAULIEU, R., Les relations de travail à l'usine, Paris, Éditions d'organisation, 1972.

SAINSAULIEU, R., L'identité du travail. Les effets culturels de l'organisation, Paris, Presses de la Fondation nationale des sciences politiques, 1977. 
SAINSAULIEU, R., Sociologie de l'organisation et de l'entreprise, Paris, Presses de la Fondation nationale des sciences politiques et Dalloz, 1987.

SAINSAULIEU, R. (dir.), L'entreprise, une affaire de société, Paris, Presses de la Fondation des sciences politiques, 1990.

SALES, A., La bourgeoisie industrielle au Québec, Montréal, PUM, 1979.

SALES, A. et BÉLANGER, N., Décideurs et gestionnaires, étude sur la direction et l'encadrement des secteurs privé et public, Québec, Conseil de la langue française, Éditeur Officiel du Québec, 1985.

SEGRESTIN, D., Le phénomène corporatiste, Paris, Fayard, 1985.

SÉGUIN, F. et CHANLAT, J.-F., L'analyse des organisations. Tome I: Les théories de l'organisation, Montréal, Gaëtan Morin, 1988.

SÉGUIN, F. et HAMEL, P.-J., "Les hauts et les bas de la profession comptable", Gestion, revue internationale de gestion, 7, 1, 1982, p. 18-24.

SÉGUIN, F. et HUBERT, H., Milieu corporatif et profession dépendante : le génie et la comptabilité, rapport de recherche, Montréal, École des HEC, 1983.

SELZNICK, P., TVA and The Grass Root, Berkeley, University of California Press, 1949.

SFEZ, I., Critique de la décision. Paris, Presses de la Fondation nationale des sciences politiques, 1976.

SERRES, M., Le passage du Nord-Ouest, Paris, Minuit, 1979.

SILVERMAN, D., La théorie des organisations, Paris. Dunod, 1973.

SIMARD, C., L'administration contre les femmes, Montréal, Boréal, 1983.

SIMARD, J. J., La longue marche des technocrates, Montréal, Saint-Martin, 1979.

SULLEROT, E. (dir.), Le fait féminin, Paris, Fayard, 1978.

SYMONS, G. (dir.), "La culture des organisations", Question de culture, no 14, Québec, Institut québécois de recherche sur la culture, 1988.

TARDIEU, H., NANCI, D. et PASCOT, P., Conception d'un système d'information, Paris, Éditions d'organisation, 1979. 
TAYLOR, J., Une organisation n'est qu'un tissu de communication, Montréal, Université de Montréal, 1988.

TESSIER, R. et TELLIER, Y., Changement planifié et développement des organisations, Sillery, Presses de l'Université du Québec, 1990.

THOENIG, J.-C., L'ère des technocrates, Paris, Éditions d'organisation, 1973.

THOENIG, J.-C. et DUPUY, F., La loi du marché, Paris, L'Harmattan, 1985.

THÉVENET, M., Audit de la culture d'entreprise, Paris, Éditions d'organisation, 1986.

TIXIER, P.-E., "Légitimité et modes de domination dans les organisations", Sociologie du travail, 30, 4, 1988, p. 615-629.

TOURAINE, A., Sociologie de l'action, Paris, Seuil, 1963.

TOURAINE, A., La société post-industrielle, Paris, Denoël-Gonthier, 1969.

TOURAINE, A., Production de la société, Paris, Seuil, 1973.

TOURAINE, A. et coll., Lutte étudiante, Paris, Seuil, 1978.

TOURAINE, A. et coll., La prophétie anti-nucléaire, Paris, Seuil, 1980.

TOURAINE, A. et coll., Solidarité, Paris, Payard, 1982.

TOURAINE, A. et coll., Le mouvement ouvrier, Paris, Fayard, 1984.

TOURAINE, A., "Existe-t-il encore une société française?" dans D. SCHNAPPER et H. MENDRAS, Six manières d'être européen, Paris, Gallimard, 1990.

TRIST, E. et BAMFORTH, K.M., "Some Social and Psychological Consequences of the Long Wall Method of Coal Getting", Human Relations, 4, 1951, p. 3-38.

VACQUIN, H., Paroles d'entreprises, Paris, Seuil, 1986.

VAILLANCOURT, J.-G., "Évolution, diversité et spécificité des associations écologiques québécoises: de la contre-culture et du conservationisme à l'environnementalisme et à l'écosocialisme" Sociologie et Sociétés, vol. XIII, no 1,1981 , p. 81-98.

VANDELAC, L., DELISLE, D. GAUTHIER, A. et PINARD, Y., Du travail et de l'amour, Montréal, Saint-Martin, 1985. 
VIENNEY, C., Socio-économie des coopératives, Paris, CIEM, 1980.

WIEVIORKA, M. et TRINH, S., Le modèle EDF, Paris, La Découverte, 1989.

ZALEZNICK, A., "Power and Politics in Organizational Life", Harvard Business Review, 48, 1970, p. 47-60.

ZALEZNICK, A. et KETS DE VRIES, M., Power and the Corporate Mind, Boston, Houghton Mifflin, 1975.

ZEY-FERRELL, M. et AIKEN, M., Complex Organization: Critical Perspectives, Glenview, Scott, Foresman \& Co., 1981. 